TRANSACTIONS OF THE

AMERICAN MATHEMATICAL SOCIETY

Volume 277, Number 1, May 1983

\title{
DISCRETE SERIES CHARACTERS AND FOURIER INVERSION ON SEMISIMPLE REAL LIE GROUPS
}

\section{BY}

REBECCA A. HERB ${ }^{1}$

\begin{abstract}
Let $G$ be a semisimple real Lie group. Explicit formulas for discrete series characters on noncompact Cartan subgroups are given. These formulas are used to give a simple formula for the Fourier transform of orbital integrals of regular semisimple orbits.
\end{abstract}

1. Introduction. Let $G$ be a connected semisimple real Lie group with finite center. Let $\hat{G}$ denote the set of equivalence classes of irreducible unitary representations of $G$. Associated with each $\pi \in \hat{G}$ is its distributional character $\theta_{\pi}$, an invariant eigendistribution on $G$. A central problem of harmonic analysis on $G$ is the Fourier inversion problem of expanding an arbitrary invariant distribution in terms of these distributional characters.

An important class of invariant distributions on $G$ arises as follows. Let $\gamma$ be a regular (semisimple) element of $G$ with centralizer $G_{\gamma}$. Let $d \dot{x}$ denote a $G$-invariant measure on the quotient $G / G_{\gamma}$, and set

$$
\Lambda_{\gamma}(f)=\int_{G / G_{\gamma}} f\left(x \gamma x^{-1}\right) d \dot{x}, \quad f \in C_{c}^{\infty}(G) .
$$

$\Lambda_{\gamma}$ is an orbital integral of the type appearing in the Selberg trace formula. $H=G_{\gamma}$ is a Cartan subgroup of $G$, and for fixed $\gamma, \Lambda_{\gamma}(f)$ is, up to a constant, the invariant integral $F_{f}^{H}(\gamma)$ defined by Harish-Chandra. The main theorem of this paper gives a Fourier inversion formula for the distribution $f \rightarrow F_{f}^{H}(\gamma)$.

Orbital integrals $\Lambda_{\gamma}$ can be defined for other elements of $G$. If $\gamma$ is semisimple, but not regular, it is known that there is a Cartan subgroup $H$ of $G$ containing $\gamma$ and a differential operator $D$ on $H$ so that

$$
\Lambda_{\gamma}(f)=\lim _{h \rightarrow \gamma} D F_{f}^{H}(h)
$$

where the limit is taken through regular elements of $H$. If $u \in G$ is unipotent, $\Lambda_{u}(f)$ can also be obtained as the derivative of an invariant integral evaluated at a singular point, although the explicit differential operators needed are only known in some cases [1]. Thus Fourier inversion formulas for $F_{f}^{H}(h), h \in H$ regular, will yield Fourier inversion formulas for many singular orbital integrals as well. In particular,

Received by the editors December 9, 1980 and, in revised form, April 5, 1982.

1980 Mathematics Subject Classification. Primary 22E30, 43A30.

${ }^{1}$ Supported in the part by NSF grant MCS 77-18723A03 
when $\gamma=1, \Lambda_{1}(f)=f(1)$ is a singular orbital integral, and the Plancherel theorem can be obtained via Fourier inversion of regular orbital integrals.

When $G$ has real rank one, Sally and Warner computed Fourier inversion formulas for $\Lambda_{\gamma}, \gamma$ semisimple (including $\gamma=1$ ) [10]. Barbasch used these results to compute Fourier inversion formulas for $\Lambda_{u}, u$ unipotent [1].

For $G$ of real rank greater than one, the methods used by Sally and Warner can be extended to obtain a Fourier inversion formula for $\Lambda_{\gamma}$ when $\gamma$ is in a dense subset of the set of regular elements [5b]. This formula is stated as Theorem 4.3 in this paper, and serves as a starting point. It contains certain complicated terms which can be interpreted as Fourier series in several variables. These series are not absolutely convergent, as the functions $F_{f}^{H}$ have jump discontinuities, and have no obvious closed form. Thus this formula gives little insight into the functions $F_{f}^{H}$ and is not suitable for applications.

In [2] and [5d,e], simple Fourier inversion formulas are obtained for certain averaged orbital integrals obtained as follows. Let $H$ be a Cartan subgroup of $G, W_{I}$ the Weyl group generated by reflections in imaginary roots of $H$. Then $W_{I}$ acts on $H$, and for regular $\gamma \in H$, the averaged orbital integral is given by

$$
\bar{\Lambda}_{\gamma}(f)=\sum_{w \in W_{l}} \Lambda_{w \gamma}(f) .
$$

In this paper, Fourier inversion formulas for $\Lambda_{\gamma}$ are obtained using those for $\bar{\Lambda}_{\gamma}$. Heuristically, the idea is as follows. Consider weighted averages

$$
\Lambda_{\gamma}^{\kappa}(f)=\sum_{w \in W_{l}} \kappa(w) \Lambda_{w \gamma}(f) \quad \text { where } \kappa(w)= \pm 1 \text { for } w \in W_{I} .
$$

For $\kappa \equiv 1, \Lambda_{\gamma}^{\kappa}=\bar{\Lambda}_{\gamma}$. For other suitable choices of $\kappa$, Shelstad proves in [12a, b, c] that $\Lambda_{\gamma}^{\kappa}$ corresponds to an ordinary averaged orbital integral for a lower-dimensional group. Thus Fourier inversion formulas for $\Lambda_{\gamma}^{\kappa}$ can be obtained as in [5d]. This is done for sufficiently many choices of $\kappa$ that the original $\Lambda_{\gamma}$ can be recovered as a sum of the $\Lambda_{\gamma}^{\kappa}$.

Theorem 1 of this paper gives a simple Fourier inversion formula for $\Lambda_{\gamma}, \gamma$ regular. The proof uses ideas of Shelstad. But rather than identify explicitly the groups and correspondences involved in [12], it is simpler to use an identity for discrete series characters which accomplishes the same purpose of reducing the problem to the study of averaged orbital integrals. This identity is stated as Theorem 2. It is the explicit form of a character identity proved by Shelstad in [12c] as a corollary of her results on orbital integrals. We give an elementary direct proof of this theorem in $\S 3$.

A more general (fewer restrictions on $G$ ), but less explicit, Fourier inversion formula has been announced by Harish-Chandra [3c].

I would like to thank Diana Shelstad for explaining to me her work on orbital integrals and suggesting its applicability to the Fourier inversion problem.

2. Statement of theorems. Let $G$ be as in $\S 1$. Let $g$ denote the Lie algebra of $G, \mathfrak{g}_{\mathbf{C}}$ its complexification. If $G_{\mathbf{C}}$ is the simply connected complex analytic group with Lie algebra $\mathfrak{g}_{\mathbf{C}}$, we assume that $G$ is the subgroup of $G_{\mathbf{C}}$ corresponding to $\mathrm{g}$. 
Let $K$ be a maximal compact subgroup of $G$ and $\theta$ the corresponding Cartan involution. Let $\mathfrak{g}=\mathfrak{f}+\mathfrak{p}$ be the Cartan decomposition of $\mathfrak{g}$ where $\mathfrak{f}$ is the Lie algebra of $K$. If $H$ is a $\theta$-stable Cartan subgroup of $G$ with Lie algebra $\mathfrak{h}$, write $\mathfrak{h}=\mathfrak{h}_{k}+\mathfrak{h}_{p}$ where $\mathfrak{h}_{k}=\mathfrak{h} \cap \mathfrak{f}, \mathfrak{h}_{p}=\mathfrak{h} \cap \mathfrak{p}$, and $H=H_{K} H_{p}$ where $H_{K}=H \cap K$ and $H_{p}=\exp \left(\mathfrak{h}_{p}\right)$. The set of roots of $\mathfrak{g}_{\mathbf{C}}$ with respect to $\mathfrak{h}_{\mathbf{C}}$ will be denoted by $\Phi=\Phi\left(\mathfrak{g}_{C}, \mathfrak{h}_{C}\right) . \Phi^{+}$denotes a choice of positive roots in $\Phi$. The subsets of $\Phi$ taking real and pure imaginary values on $\mathfrak{h}$ will be denoted by $\Phi_{R}=\Phi_{R}(\mathfrak{g}, \mathfrak{h})$ and $\Phi_{I}=\Phi_{I}(\mathfrak{g}, \mathfrak{h})$, respectively. Let $r_{I}(H)=\frac{1}{2}\left[\Phi_{I}\right]$. For any root system $\Phi, L(\Phi)$ denotes the weight lattice and $W(\Phi)$ the Weyl group of $\Phi$.

As in [5e], for any root system $\Phi$ spanned by strongly orthogonal roots, we say a root system $\varphi \subseteq \Phi$ is a two-structure for $\Phi$ if: (i) all simple factors of $\varphi$ are of type $A_{1}$ or $B_{2} \cong C_{2}$; (ii) if $\varphi^{+}$is any set of positive roots for $\varphi$ then $\left\{w \in W(\Phi) \mid w \varphi^{+}=\right.$ $\varphi^{+}$\} contains no elements of determinant -1 .

Let $\sigma(\Phi)$ denote the set of all two-structures for $\Phi$. All elements of $\sigma(\Phi)$ are conjugate under $W(\Phi)$. If $\Phi^{+}$is a choice of positive roots for $\Phi$, let $\varphi^{+}=\varphi \cap \Phi^{+}$ for any $\varphi \in \mathcal{T}(\Phi)$. In [5e] a distinguished element $\varphi_{0} \in \mathcal{T}(\Phi)$ is chosen for each type of root system. Then each $\varphi \in \mathcal{T}(\Phi)$ is assigned a sign $\varepsilon\left(\varphi: \Phi^{+}\right)=\operatorname{det} \sigma$ where $\sigma$ is an element of $W(\Phi)$ satisfying $\sigma \varphi_{0}^{+}=\varphi^{+}$. Because of (iii) above, det $\sigma$ is well defined even though $\sigma$ may not be unique. Alternatively, the signs $\varepsilon\left(\varphi: \Phi^{+}\right)$are uniquely determined by:

(i) for all $\varphi \in \mathcal{T}(\Phi)$ and $\sigma \in W$ such that $\sigma \varphi^{+} \subseteq \Phi^{+}$,

$$
\varepsilon\left(\sigma \varphi: \Phi^{+}\right)=\operatorname{det} \sigma \varepsilon\left(\varphi: \Phi^{+}\right)
$$

(ii) $\Sigma_{\varphi \in \mathcal{T}(\Phi)} \varepsilon\left(\varphi: \Phi^{+}\right)=1$.

If $\Phi$ is simple, $\mathcal{T}(\Phi)$ will consist of all root systems $\varphi$ contained in $\Phi$ of type given by the following table which also gives the values of $[\mathcal{T}(\Phi)]$ and $[L(\varphi): L(\Phi)]$.

\begin{tabular}{|c|c|c|c|}
\hline$\Phi$ & $\varphi$ & {$[\widetilde{T}(\varphi)]$} & {$[L(\varphi): L(\Phi)]$} \\
$A_{1}$ & $A_{1}$ & 1 & 1 \\
$B_{2 n}$ & $B_{2}^{n}$ & $(2 n) ! / n ! 2^{n}$ & $2^{n-1}$ \\
$B_{2 n+1}$ & $B_{2}^{n} \times B_{1}$ & $(2 n+1) ! / n ! 2^{n}$ & $2^{n}$ \\
$C_{2 n}$ & $C_{2}^{n}$ & $(2 n) ! / n ! 2^{n}$ & 1 \\
$C_{2 n+1}$ & $C_{2}^{n} \times C_{1}$ & $(2 n+1) ! / n ! 2^{n}$ & 1 \\
$D_{2 n}$ & $A_{1}^{2 n}$ & $(2 n) ! / n ! 2^{n}$ & $2^{n-1}$ \\
$E_{7}$ & $A_{1}^{7}$ & $5 \times 3^{3}$ & $2^{3}$ \\
$E_{8}$ & $A_{1}^{8}$ & $5^{2} \times 3^{4}$ & $2^{4}$ \\
$F_{4}$ & $B_{2}^{2}$ & $3^{2}$ & 2 \\
$G_{2}$ & $A_{1}^{2}$ & 3 & 2 \\
\hline
\end{tabular}

Let $W(G, H)=N_{G}(H) / H$ where $N_{G}(H)$ is the normalizer in $G$ of $H$. For each $\alpha \in \Phi_{R}$, let $H_{\alpha} \in \mathfrak{h}_{p}$ be dual to $\check{\alpha}=2 \alpha /\langle\alpha, \alpha\rangle$ under the killing form. Let $\gamma_{\alpha}=$ $\exp \left(\pi i H_{\alpha}\right) \in H_{\mathbf{C}}$. Then $\left\{\gamma_{\alpha} \mid \alpha \in \Phi_{R}\right\}$ generate the subgroup $Z\left(\mathfrak{h}_{p}\right)=K \cap \exp \left(i \mathfrak{h}_{p}\right)$ of $H_{K}$. 
Let $L_{H}=C_{G}\left(H_{p}\right)$, the centralizer in $G$ of $H_{p}$, and write $L_{H}=M_{H} H_{p}$ in its Langlands decomposition. Then $M=M_{H}$ is a reductive group with compact Cartan subgroup $H_{K}$. Let $\operatorname{Car}(M)$ denote a full set of $\theta$-stable representatives of $M$-conjugacy classes of Cartan subgroups of $M$. These representatives can be chosen so that for $J \in \operatorname{Car}(M), J_{K}^{0} \subseteq H_{K}$. Each $J \in \operatorname{Car}(M)$ corresponds to a set $R$ of strongly orthogonal singular imaginary roots in $\Phi_{I}^{+}(\mathfrak{g}, \mathfrak{h})$. Let $\nu$ be the corresponding Cayley transform. Then $\nu\left(\mathfrak{h}_{k}\right)=\dot{\mathrm{i}}_{k}+i \mathrm{i}_{p}$ and $\nu R$ spans $\Phi_{R}^{+}(\mathrm{m}, \dot{\mathrm{j}})$. For each $J \in \operatorname{Car}(M), \tilde{J}=J H_{p}$ is a Cartan subgroup of $G$. There may be distinct elements $J, J^{\prime} \in \operatorname{Car}(M)$ with $\tilde{J}$ conjugate to $\tilde{J}^{\prime}$ in $G$.

The unitary character group of $H$ will be parameterized by pairs $\left(b^{*}, \mu\right)$ where $b^{*} \in \hat{H}_{k}$ and $\mu \in \mathfrak{h}_{p}^{*}$, the real dual of $\mathfrak{h}_{p}$. To each such pair $\left(b^{*}, \mu\right)$ there corresponds a tempered invariant eigendistribution $\theta\left(H, b^{*}, \mu\right)$ on $G$ defined as in [5b]. If $b^{*} \in \hat{H}_{k}^{\prime}=\left\{b^{*} \in \hat{H}_{k} \mid w b^{*} \neq b^{*}\right.$ for $\left.w \in W\left(\Phi_{I}\right), w \neq 1\right\}$, then $\theta\left(H, b^{*}, \mu\right)$ is, up to a sign, the character of a tempered unitary representation of $G$ induced from a parabolic subgroup of $G$ with split part $H_{p}$. If $b^{*}$ is not regular, $\theta\left(H, b^{*}, \mu\right)$ also has a character-theoretic interpretation given in [6].

Let $G^{\prime}$ be the set of regular semisimple elements of $G$ and let $H^{\prime}=H \cap G^{\prime}$. For $h \in H^{\prime}, f \in C_{c}^{\infty}(G)$, define

$$
F_{f}^{H}(h)=\varepsilon_{R}(h) \Delta(h) \int_{G / H} f\left(x h x^{-1}\right) d \dot{x}
$$

where $\varepsilon_{R}(h)$ and $\Delta(h)$ are defined as in [14]. Let $\Phi^{+}\left(g_{\mathbf{C}}, \mathfrak{h}_{\mathbf{C}}\right)$ be the set of positive roots inherent in the definition of $\Delta$. Assume all Haar measures are normalized as in [5b] and let $\operatorname{vol}\left(H_{K}\right)$ denote the total mass of $H_{K}$.

THEOREM 1. Let $f \in C_{c}^{\infty}(G)$ and let $h_{k} h_{p} \in H^{\prime}$ where $h_{k} \in H_{K}$ and $h_{p} \in H_{p}$. Then

$$
\begin{aligned}
F_{f}^{H}\left(h_{k} h_{p}\right)= & \operatorname{vol}\left(H_{K}\right)^{-1}(2 \pi)^{-\operatorname{dim} \mathfrak{h}_{p}} \\
& \times \sum_{J \in \operatorname{Car}(M)}\left(\frac{i}{2}\right)^{\operatorname{dim} i_{p}} \frac{(-1)^{r_{f}(J)}}{[W(M, J)]\left[Z\left(\mathfrak{h}_{p}\right) \cap Z\left(\dot{\mathrm{j}}_{p}\right)\right]} \\
& \times \sum_{b^{*} \in \hat{J}_{K}} \int_{\mathfrak{h}_{p}^{*}} h_{p}^{-i \mu^{\prime}} \int_{\mathrm{i}_{p}^{*}} \theta\left(J H_{p}, b^{*}, \mu^{\prime} \otimes \mu\right)(f) \\
& \times \sum_{w \in W\left(M, H_{K}\right)} \operatorname{det} w K\left(M, J, b^{*}, \mu, w h_{k}\right) d \mu d \mu^{\prime} .
\end{aligned}
$$

Let $J \in \operatorname{Car}(M)$, and let $R$ be the corresponding set of strongly orthogonal singular imaginary roots of $\left(\mathfrak{m}, \mathfrak{h}_{k}\right), \nu$ the Cayley transform. Fix $b^{*} \in \hat{J}_{K}, \mu \in j_{p}^{*}$, and $h_{k} \in H_{K} \cap M^{\prime}$. Then $K\left(M, J, b^{*}, \mu, h_{k}\right)$ is defined as follows. Let $\Phi=\Phi_{R}(\mathrm{~m}, \mathrm{i})$ and $\Phi^{+}=\Phi \cap \nu \Phi^{+}\left(g_{C}, \mathfrak{h}_{\mathrm{C}}\right)$. Let $\varphi_{R}$ be a two-structure for $\Phi$ with $\nu R \subseteq \varphi_{R}$.

Decompose $\varphi_{R}=\varphi_{1} \cup \cdots \cup \varphi_{k}$ where the $\varphi_{j}, 1 \leqslant j \leqslant k$, are simple root systems. Write $\dot{\mathfrak{l}}_{p}=a_{1} \oplus \cdots \oplus \mathfrak{a}_{k}$ where $1 \leqslant j \leqslant k, a_{j}=\Sigma_{\alpha \in \varphi_{j}} \mathbf{R} H_{\alpha}$. Then $\nu h_{k}$ can be decomposed as $\nu h_{k}=j_{0} a_{1} \cdots a_{k}$ where $j_{0} \in J_{K}$ and $a_{j} \in \exp \left(i a_{j}\right), 1 \leqslant j \leqslant k$. Let 
$\mu_{j} \in \mathfrak{a}_{j}^{*}$ be the restriction of $\mu$ to $a_{j}, b_{j}^{*}$ the restriction of $b^{*}$ to $Z\left(a_{j}\right)$. Then

$$
K\left(M, J, b^{*}, \mu, h_{K}\right)=\frac{\varepsilon\left(\varphi_{R}: \Phi^{+}\right)[\mathcal{T}(\Phi)]}{\left[L\left(\varphi_{R}\right): L(\Phi)\right]} \overline{b^{*}\left(j_{0}\right)} \prod_{j=1}^{k} K\left(\varphi_{j}^{+}, b_{j}^{*}, \mu_{j}, a_{j}\right)
$$

is a product of factors corresponding to the simple root systems $\varphi_{j}$.

Let $\varphi=\varphi_{j}$ be one of these and write $a=a_{j}, \varphi^{+}=\varphi \cap \Phi^{+}$. Suppose $\varphi$ is of type $A_{1}$ and $\varphi^{+}=\{\alpha\}$. Write $a \in \exp (i a)^{\prime}$ as $a=\exp \left(-i \theta H_{\alpha}\right)$ where $0<|\theta|<\pi$. Each $\mu \in \mathfrak{a}^{*}$ is determined by $\mu\left(H_{\alpha}\right)$ which we also denote by $\mu$. Define

$$
S\left(\varphi^{+}, \mu, a\right)=\frac{2}{\|\alpha\|} \frac{\sinh \mu(\theta \mp \pi)}{\sinh \pi \mu}
$$

where

$$
\theta \mp \pi= \begin{cases}\theta-\pi & \text { if } 0<\theta<\pi \\ \theta+\pi & \text { if }-\pi<\theta<0 .\end{cases}
$$

Then for $b^{*} \in Z(\mathfrak{a}) \hat{\text {, }}$

$$
K\left(\varphi^{+}, b^{*}, \mu, a\right)=S\left(\varphi^{+}, \mu, a\right)+b^{*}\left(\gamma_{\alpha}\right) S\left(\varphi^{+}, \mu, \gamma_{\alpha} a\right) .
$$

Suppose $\varphi$ is of type $B_{2}$ and $\varphi^{+}=\left\{\alpha_{1}, \alpha_{2}, \beta_{1}, \beta_{2}\right\}$ where $\beta_{1}=\frac{1}{2}\left(\alpha_{1}+\alpha_{2}\right)$ and $\beta_{2}=\frac{1}{2}\left(\alpha_{1}-\alpha_{2}\right)$. Then exactly one of $\nu^{-1} \beta_{i}, i=1,2$, is compact. Write

$$
\varepsilon\left(\varphi^{+}\right)=\left\{\begin{array}{cl}
1 & \text { if } \nu^{-1} \beta_{2} \text { is compact } \\
-1 & \text { if } \nu^{-1} \beta_{1} \text { is compact }
\end{array}\right.
$$

Then $a \in \exp (i \mathfrak{a})^{\prime}$ can be written as $a=\exp \left(-i \sum_{j=1}^{2} \theta_{j} H_{\alpha_{j}}\right)$ where $0<\left|\theta_{j}\right|<\pi, j=1$, 2 , and $\left|\theta_{1}\right| \neq\left|\theta_{2}\right|$. For $\mu \in a^{*}$ write $\mu_{i}=\mu\left(H_{\alpha_{i}}\right)$. Define

$S\left(\varphi^{+}, \mu, a\right)$

$$
=\frac{4}{\left\|\alpha_{1}\right\|^{2}} \times \begin{cases}\frac{\sinh \mu_{1} \theta_{1} \sinh \mu_{2}\left(\theta_{2} \mp \pi\right)}{\sinh \pi \mu_{1} \sinh \pi\left(\mu_{1}+\mu_{2}\right)} & \text { if }\left|\theta_{1}\right|<\left|\theta_{2}\right|, \\ \frac{\sinh \mu_{1}\left(\theta_{1} \mp \pi\right) \sinh \mu_{2}\left(\theta_{2} \mp\left(1+\mu_{1} \mu_{2}^{-1}\right) \pi\right)}{\sinh \pi \mu_{1} \sinh \pi\left(\mu_{1}+\mu_{2}\right)} & \text { if }\left|\theta_{1}\right|>\left|\theta_{2}\right| .\end{cases}
$$

Write $\varphi_{l}^{+}=\left\{\alpha_{1}, \alpha_{2}\right\}$ and $\varphi_{s}^{+}=\left\{\beta_{1}, \beta_{2}\right\}$. Define

$$
S\left(\varphi_{l}^{+}, \mu, a\right)=\frac{4}{\left\|\alpha_{1}\right\|^{2}} \frac{\sinh \mu_{1}\left(\theta_{1} \mp \pi\right) \sinh \mu_{2}\left(\theta_{2} \mp \pi\right)}{\sinh \pi \mu_{1} \sinh \pi \mu_{2}}
$$

and

$$
\begin{aligned}
& S\left(\varphi_{s}^{+}, \mu, a\right) \\
& =\frac{4 \varepsilon\left(\varphi^{+}\right)}{\left\|\beta_{1}\right\|^{2}}\left[\frac{\sinh \left(\mu_{1}+\mu_{2}\right)\left(\left(\theta_{1}+\theta_{2}\right) / 2 \mp \pi\right) \sinh \left(\mu_{1}-\mu_{2}\right)\left(\left(\theta_{1}-\theta_{2}\right) / 2 \mp \pi\right)}{\sinh \pi\left(\mu_{1}+\mu_{2}\right) \sinh \pi\left(\mu_{1}-\mu_{2}\right)}\right. \\
& \left.+\frac{\sinh \left(\mu_{1}+\mu_{2}\right)\left(\left(\theta_{1}+\theta_{2}\right) / 2\right) \sinh \left(\mu_{1}-\mu_{2}\right)\left(\left(\theta_{1}-\theta_{2}\right) / 2\right)}{\sinh \pi\left(\mu_{1}+\mu_{2}\right) \sinh \pi\left(\mu_{1}-\mu_{2}\right)}\right] .
\end{aligned}
$$


Then for $b^{*} \in Z(a)$,

$$
\begin{aligned}
K\left(\varphi^{+}, \mu, b^{*}, a\right)= & -2 S\left(\varphi^{+}, \mu, a\right)+\frac{1}{2} S\left(\varphi_{s}^{+}, \mu, a\right) \\
& +b^{*}\left(\gamma_{\beta_{1}}\right)\left\{-2 S\left(\varphi^{+}, \mu, \gamma_{\beta_{1}} a\right)+\frac{1}{2} S\left(\varphi_{s}^{+}, \mu, \gamma_{\beta_{1}} a\right)\right\} \\
& +b^{*}\left(\gamma_{\alpha_{1}}\right) 2 S\left(\varphi_{l}^{+}, \mu, \gamma_{\alpha_{1}} a\right) .
\end{aligned}
$$

Theorem 1 will be obtained by simplifying the formula for $F_{f}^{H}$ given in Theorem 4.3. The main result needed is an identity involving constants which appear in discrete series character formulas. Let $\Phi$ be a root system spanned by strongly orthogonal roots, $\Phi^{+}$a choice of positive roots. We assume $\Phi$ comes equipped with a subset $\Phi_{C P T}$ called the set of compact roots. Let $L=L(\Phi), W=W(\Phi)$, and $W_{K}=W\left(\Phi_{C P T}\right)$. For each $\tau \in L, w \in W$, there is a constant $c\left(w: \tau: \Phi^{+}\right)$which arises as follows.

Let $G$ be a split real group satisfying our hypotheses with root system of type $\Phi$. Then $G$ has a compact Cartan subgroup $T$ with Lie algebra $\mathrm{t}$, and $L$ can be identified with the lattice of elements $\tau \in i$ t $^{*}$ for which $\xi_{\tau}(\exp H)=\exp (\tau(H))$, $H \in \mathrm{t}_{\mathrm{C}}$, gives a well-defined character of $T_{\mathrm{C}}$. We assume that when $\Phi$ is identified with $\Phi\left(\mathrm{g}_{C}, \mathrm{t}_{\mathbf{C}}\right), \Phi_{C P T}$ corresponds to the set of compact roots of $\mathrm{t}$. Corresponding to each $\tau \in L$ there is $\lambda$ invariant eigendistribution $\theta_{\tau}$ defined by Harish-Chandra in [3a] and [3b]. If $\tau \in L^{\prime}=\left\{\tau \in L \mid \Pi_{\alpha \in \Phi}\langle\alpha, \tau\rangle \neq 0\right\}, \theta_{\tau}$ is, up to sign, the character of a discrete series representation of $G$.

Let $A$ be a split Cartan subgroup of $G$ with Lie algebra $\mathfrak{a} \subseteq \mathfrak{p}$. Let $\nu$ be a Cayley transform satisfying $\nu \mathrm{t}=i \mathrm{a}$. Let $\mathrm{a}^{+}=\left\{H \in a \mid \nu \alpha(H)>0\right.$ for all $\left.\alpha \in \Phi^{+}\right\}$. Then for $h \in A^{+}=\exp \left(\mathrm{a}^{+}\right)$,

$$
\theta_{\tau}(h)=\Delta(h)^{-1} \sum_{w \in W} \operatorname{det} w c\left(w: \tau: \Phi^{+}\right) \xi_{w \tau}\left(\nu^{-1} h\right) .
$$

The constant $c\left(w: w^{-1} \tau: \Phi^{+}\right)$depends only on the coset of $W$ in $W / W_{K}$ and we define

$$
\bar{c}\left(\tau: \Phi^{+}\right)=\sum_{w \in W / W_{K}} c\left(w: w^{-1} \tau: \Phi^{+}\right) .
$$

Then it is proved in $[\mathbf{5 c}, \mathbf{e}]$ that

$$
\bar{c}\left(\tau: \Phi^{+}\right)=\sum_{\varphi \in \mathscr{T}(\Phi)} \varepsilon\left(\varphi: \Phi^{+}\right) \bar{c}\left(\tau: \varphi^{+}\right) .
$$

Since all simple factors of each two-structure $\varphi$ for $\Phi$ are of type $A_{1}$ or $B_{2}, \bar{c}\left(\tau: \varphi^{+}\right)$ can be evaluated explicitly as in [5c]. Theorem 2 gives a formula for $c\left(w: \tau: \Phi^{+}\right)$in terms of averaged constants $\bar{c}\left(w \tau: \Phi^{+}(\lambda)\right)$ where the $\Phi(\lambda)$ are root systems contained in $\Phi$.

Using reduction procedures described by Harish-Chandra in [3a], knowledge of the constants $c\left(w: \tau: \Phi^{+}\right)$is sufficient to determine discrete series character formulas on any Cartan subgroup of any group $G$ satisfying our assumptions. 
Let $\Lambda=\Lambda(\Phi)$ denote the root lattice of $\Phi$. For $\lambda \in \Lambda$, let $\Phi(\lambda)=\{\beta \in \Phi \mid$ $\langle\beta, \lambda\rangle /\langle\beta, \beta\rangle \in Z\}$. Then for $\lambda_{1}, \lambda_{2} \in \Lambda, \Phi\left(\lambda_{1}\right)=\Phi\left(\lambda_{2}\right)$ if and only if $\lambda_{1}-\lambda_{2}$ $\in \Lambda_{0}=\{\lambda \in \Lambda \mid\langle\lambda, \beta\rangle /\langle\beta, \beta\rangle \in Z$ for all $\beta \in \Phi\}$. Define a homomorphism $\chi$ : $\Lambda \rightarrow Z / 2 Z$ by setting

$$
\chi(\alpha)= \begin{cases}0 & \text { if } \alpha \in \Phi_{C P T}, \\ 1 & \text { if } \alpha \notin \Phi_{C P T}\end{cases}
$$

and extending linearly. This extension is well defined because if $\alpha, \beta$, and $\alpha+\beta$ are all roots, then $\chi(\alpha+\beta) \equiv \chi(\alpha)+\chi(\beta)(\bmod 2)$. For $\lambda_{1}, \lambda_{2} \in \Lambda$, define $\lambda_{1} \equiv \lambda_{2}$ if $\chi\left(\lambda_{1}-\lambda_{2}\right)=0$. Then for $\lambda \in \Lambda$ and $w \in W(\Phi)$ we define

$$
\kappa_{\lambda}(w)=\left\{\begin{array}{cl}
1 & \text { if } w^{-1} \lambda \equiv \lambda, \\
-1 & \text { if } w^{-1} \lambda \neq \lambda .
\end{array}\right.
$$

It is easy to check from the definitions that $\kappa_{\lambda}$ depends only on the coset of $\lambda$ in $\Lambda / \Lambda_{0}$ and of $w$ in $W / W_{K}$.

Suppose $\Phi$ is simple. Then the root systems $\Phi(\lambda), \lambda \in \Lambda$, will be all root systems $\Phi(\lambda) \subseteq \Phi$ of type given by the following table.

\begin{tabular}{|l|l|}
\hline$\Phi$ & $\Phi(\lambda)$ \\
$A_{1}$ & $A_{1}$ \\
$B_{n}$ & $B_{p} \times B_{n-p}, 0 \leqslant p \leqslant n$ \\
$C_{n}$ & $D_{2 p} \times C_{n-2 p}, 0 \leqslant 2 p \leqslant n$ \\
$D_{2 n}$ & $D_{2 p} \times D_{2(n-p)}, 0 \leqslant p \leqslant n$ \\
$E_{7}$ & $E_{7}, D_{6} \times A_{1}$ \\
$E_{8}$ & $E_{8}, E_{7} \times A_{1}, D_{8}$ \\
$F_{4}$ & $F_{4}, B_{3} \times B_{1}, C_{4}$ \\
$G_{2}$ & $G_{2}, A_{1}^{2}$ \\
\hline
\end{tabular}

We note that when $\Phi$ is identified with $\Phi\left(g_{\mathbf{C}}, t_{\mathbf{C}}\right)$ as above, then $\left\{\kappa_{\lambda} \mid \lambda \in \Lambda\right\}$ is a subset of the set of "characters" $\kappa$ of $\mathscr{D}(T)$ considered by Shelstad in [12a] and that $\Phi(\lambda)$ is the root system of the endoscopic group $H$ associated to $\kappa_{\lambda}$. The characters $\kappa$ which are of the form $\kappa_{\lambda}, \lambda \in \Lambda$, are exactly those for which $H$ "shares" both $T$ and $A$ with $G$.

Given a fixed choice $\Phi^{+}$of positive roots for $\Phi$, we will describe in $\S 3$ a way of assigning to each $\lambda \in \Lambda$ a sign $\varepsilon\left(\lambda: \Phi^{+}\right)$. For each $\lambda \in \Lambda$, let $\Phi^{+}(\lambda)=\Phi(\lambda) \cap \Phi^{+}$.

THEOREM 2. Let $n=\operatorname{rank} \Phi$. Then for $w \in W, \tau \in L$,

$$
c\left(w: \tau: \Phi^{+}\right)=2^{-n} \sum_{\lambda \in \Lambda / \Lambda_{0}} \varepsilon\left(\lambda: \Phi^{+}\right) \kappa_{\lambda}(w) \bar{c}\left(w \tau: \Phi^{+}(\lambda)\right) .
$$

Combining Theorem 2 with (2.1) and known values for $\bar{c}\left(\tau: \Phi^{+}\right)$when $\Phi$ is of type $A_{1}$ or $B_{2}$ gives a complete description of the constants $c\left(w: \tau: \Phi^{+}\right)$. Much simpler formulas for these constants have been obtained in special cases, for example when the corresponding discrete series representation is holomorphic. Our formula can be simplified to agree with the formula of Hecht [4] in this case. 
Although complicated, the formulas of Theorem 2 and (2.1) are well suited to simplifying the terms occurring in the Fourier inversion formula for $F_{f}{ }^{H}$. For other discrete series character formulas the reader is referred to work of Hirai [7], Schmid [11], Martens [8], Midorikawa [9], and Vargas [13].

\section{Proof of Theorem 2.}

Lemma 3.1. Let $\lambda \in \Lambda$ and $s \in W(\Phi(\lambda))$. Then for all $w \in W, \kappa_{\lambda}(s w)=\kappa_{\lambda}(w)$.

Proof. It is enough to prove that for all $\alpha \in \Phi(\lambda)$ and $w \in W, \kappa_{\lambda}\left(s_{\alpha} w\right)=\kappa_{\lambda}(w)$ where $s_{\alpha}$ denotes the reflection corresponding to $\alpha$. But $w^{-1} s_{\alpha} \lambda=w^{-1} \lambda-$ $2(\langle\alpha, \lambda\rangle /\langle\alpha, \alpha\rangle) w^{-1} \alpha \equiv w^{-1} \lambda$ since $\langle\alpha, \lambda\rangle /\langle\alpha, \alpha\rangle \in Z$ and $2 n \beta \equiv 0$ for any $n \in Z$, $\beta \in \Phi$. The result follows from the definition of $\kappa_{\lambda}$.

LEMma 3.2. Let $\lambda \in \Lambda, v, w \in W$. Then $\kappa_{v \lambda}(w)=\kappa_{\lambda}\left(v^{-1}\right) \kappa_{\lambda}\left(v^{-1} w\right)$.

Proof. This follows trivially from the definition of $\kappa_{\lambda}$.

We note that the above two lemmas appear in [12a] as Propositions 3.1 and 3.3.

We will now assign to each $\lambda \in \Lambda$ and system $\Phi^{+}$of positive roots for $\Phi$ a sign $\varepsilon\left(\lambda: \Phi^{+}\right)$. We will first do this for positive systems chosen as follows.

(3.3) If $\Phi$ is of type $A_{1}, B_{2 n+1}, D_{2 n}, E_{7}, E_{8}$, or $G_{2}$, pick $\Phi^{+}$so that all simple roots are noncompact. If $\Phi$ is of type $B_{2 n}, C_{n}$, or $F_{4}$, pick $\Phi^{+}$so that all long simple roots are noncompact and all short simple roots are compact. If $\Phi$ is not simple, pick positive roots for each simple factor as above.

For $\Phi^{+}$chosen as above, if $\Phi$ is of type $A_{1}, B_{n}, D_{2 n}, E_{7}, E_{8}$, or $G_{2}$, set $\varepsilon\left(\lambda: \Phi^{+}\right)=1$ for all $\lambda \in \Lambda$. For $\Phi$ of type $C_{n}$, denote the simple roots for $\Phi^{+}$by $\left\{\alpha_{1}, \ldots, \alpha_{n}\right\}$ when $\left\langle\alpha_{i}, \alpha_{i+1}\right\rangle=-1,1 \leqslant i \leqslant n-1$, and $\left\langle\alpha_{n-1}, \alpha_{n}\right\rangle=-2$. Let $\Lambda_{1}=$ $\left\{\alpha_{1}+\alpha_{3}+\cdots+\alpha_{2 p-1} \mid 0 \leqslant p \leqslant k\right\}$ where $n=2 k$ or $2 k+1$. If $\Phi$ is of type $F_{4}$, let $\Lambda_{1}=\left\{0, \alpha_{1}, \alpha_{2}\right\}$ where $\alpha_{1}$ is a short simple root and $\alpha_{2}$ is a long simple root for $\Phi^{+}$. In either case, for $\lambda \in \Lambda_{1}$, define $W\left(\lambda: \Phi^{+}\right)=\left\{\sigma \in W \mid \sigma \Phi^{+}(\lambda) \subseteq \Phi^{+}\right\}$. Then for any $\lambda \in \Lambda$ there are $\lambda_{1} \in \Lambda_{1}$ and $\sigma \in W\left(\lambda_{1}: \Phi^{+}\right)$with $\Phi^{+}(\lambda)=\sigma \Phi^{+}\left(\lambda_{1}\right)$. Define $\varepsilon\left(\lambda: \Phi^{+}\right)=\operatorname{det} \sigma \kappa_{\lambda_{1}}\left(\sigma^{-1}\right)$. For $\Phi$ of type $F_{4}, \lambda_{1}$ and $\sigma$ are uniquely determined by $\lambda$. For $\Phi$ of type $C_{n}, \lambda_{1}$ is uniquely determined by $\lambda$. Each element of $W\left(\lambda_{1}: \Phi^{+}\right)$is of the form $\sigma=v s$ where $v \in W_{K} \cap W\left(\lambda_{1}: \Phi^{+}\right)$and $s \in\left\{\sigma \in W \mid \sigma \Phi^{+}\left(\lambda_{1}\right)=\Phi^{+}\right.$ $\left.\left(\lambda_{1}\right)\right\}$. Again, $v$ is uniquely determined by $\lambda$ but $s$ is not. However, $\operatorname{det} \sigma \kappa_{\lambda_{1}}\left(\sigma^{-1}\right)=$ det $v$ is well defined.

If $\Phi$ is not simple, write $\Phi^{+}=\Phi_{1}^{+} \cup \cdots \cup \Phi_{s}^{+}$where the $\Phi_{i}$ are simple. Then for $\lambda_{i} \in \Lambda\left(\Phi_{i}\right), 1 \leqslant i \leqslant s$, define $\varepsilon\left(\lambda_{1}+\cdots+\lambda_{s}: \Phi^{+}\right)=\prod_{i=1}^{s} \varepsilon\left(\lambda_{i}: \Phi_{i}^{+}\right)$.

If $\Phi^{+}$is chosen as in (3.3) and $v \in W$, define $\varepsilon\left(\lambda: v \Phi^{+}\right)=\kappa_{\lambda}(v) \varepsilon\left(v^{-1} \lambda: \Phi^{+}\right)$for all $\lambda \in \Lambda$.

LEMMA 3.4. Let $\lambda \in \Lambda$ and $\sigma \in W\left(\lambda: \Phi^{+}\right)=\left\{\sigma \in W \mid \sigma \Phi^{+}(\lambda) \subseteq \Phi^{+}\right\}$. Then for all $w \in W, \varepsilon\left(\sigma \lambda: \Phi^{+}\right) \kappa_{\sigma \lambda}(w)=\operatorname{det} \sigma \varepsilon\left(\lambda: \Phi^{+}\right) \kappa_{\lambda}\left(\sigma^{-1} w\right)$.

Proof. Using (3.2), it is enough to prove that $\varepsilon\left(\sigma \lambda: \Phi^{+}\right)=\kappa_{\lambda}\left(\sigma^{-1}\right) \operatorname{det} \sigma \varepsilon\left(\lambda: \Phi^{+}\right)$ for all $\sigma \in W\left(\lambda: \Phi^{+}\right)$. Suppose first that $\Phi^{+}$has been chosen as in (3.3). Write $\varepsilon\left(\lambda: \Phi^{+}\right)=\varepsilon(\lambda)$. If $\Phi$ is of type $C_{n}$ or $F_{4}$ and $\lambda \in \Lambda_{1}$, then (3.4) follows directly from the definition of $\varepsilon(\sigma \lambda)$. If $\lambda$ is arbitrary, then $\Phi^{+}(\lambda)=\tau \Phi^{+}\left(\lambda_{1}\right)$ for some 
$\lambda_{1} \in \Lambda_{1}$ and $\tau \in W\left(\lambda_{1}, \Phi^{+}\right)$. If $\sigma \in W\left(\lambda: \Phi^{+}\right)$, then $\sigma \tau \in W\left(\lambda_{1}: \Phi^{+}\right)$and the result follows from using (3.2) and the definitions of $\varepsilon\left(\sigma \tau \lambda_{1}\right)$ and $\varepsilon\left(\tau \lambda_{1}\right)$.

If $\Phi$ is of type $A_{1}, B_{n}, D_{n}, E_{7}, E_{8}$, or $G_{2}, \varepsilon(\lambda)=1$ for all $\lambda$ so we must show that $\kappa_{\lambda}\left(\sigma^{-1}\right)=\operatorname{det} \sigma$ for all $\sigma \in W\left(\lambda: \Phi^{+}\right)$. Write $\sigma \in W\left(\lambda: \Phi^{+}\right)$as $\sigma=s_{\alpha_{1}} \cdots s_{\alpha_{k}}$ where the $\alpha_{i}$ are simple roots and $k$ is minimal. In this case each $\alpha_{i}$ is noncompact. The proof is by induction on $k=l(\sigma)$.

If $k=0$ the result is obvious. Suppose $l(\sigma)=k \geqslant 1$. Because $s_{\alpha_{1}} \cdots s_{\alpha_{k}}$ is a reduced expression for $\sigma, \sigma_{0}=s_{\alpha_{2}} \cdots s_{\alpha_{k}} \in W\left(\lambda: \Phi^{+}\right)$also and $s_{\alpha_{1}} \in W\left(\sigma_{0} \lambda: \Phi^{+}\right)$. Using (3.2) and the induction hypothesis, $\kappa_{\lambda}\left(\sigma^{-1}\right)=\kappa_{\sigma_{0} \lambda}\left(s_{\alpha_{1}}\right) \kappa_{\lambda}\left(\sigma_{0}^{-1}\right)=$ $\operatorname{det} \sigma_{0} \kappa_{\sigma_{0} \lambda}\left(s_{\alpha_{1}}\right)$. Further $s_{\alpha_{1}} \Phi^{+}\left(\sigma_{0} \lambda\right) \subseteq \Phi^{+}$implies that $\alpha_{1} \notin \Phi\left(\sigma_{0} \lambda\right)$ so that $2\left(\left\langle\sigma_{0} \lambda, \alpha_{1}\right\rangle /\left\langle\alpha_{1}, \alpha_{1}\right\rangle\right) \alpha_{1} \neq 0$ and $s_{\alpha_{1}} \sigma_{0} \lambda Z \sigma_{0} \lambda$. Thus $\kappa_{\sigma_{0} \lambda}\left(s_{\alpha_{1}}\right) \operatorname{det} \sigma_{0}=-\operatorname{det} \sigma_{0}=$ $\operatorname{det} \sigma$.

If $v \Phi^{+}$is another choice of positive roots, $v^{-1} W\left(\lambda: v \Phi^{+}\right) v=W\left(v^{-1} \lambda: \Phi^{+}\right)$and the result follows by using the first case, (3.2), and the definition of $\varepsilon\left(\lambda: v \Phi^{+}\right)$.

TheOREM 2. Let $w \in W, \tau \in L$. Let $n=\operatorname{rank} \Phi$. Then

$$
c\left(w: \tau: \Phi^{+}\right)=2^{-n} \sum_{\lambda \in \Lambda / \Lambda_{0}} \varepsilon\left(\lambda: \Phi^{+}\right) \kappa_{\lambda}(w) \bar{c}\left(w \tau: \Phi^{+}(\lambda)\right) .
$$

Before proceeding with the proof of Theorem 2, we will illustrate it in the cases $n=1,2$, where all values of $c\left(w: \tau: \Phi^{+}\right)$are known [5a]. Note that in these cases all the signs $\varepsilon\left(\lambda: \Phi^{+}\right)$are one.

Case I. Suppose $\Phi^{+}=\{\alpha\}$ is of type $A_{1}$. Then for $w \in W, \tau \in L$,

$$
c\left(w: w^{-1} \tau: \Phi^{+}\right)= \begin{cases}1 & \text { if }\langle\alpha, \tau\rangle<0 \\ 0 & \text { otherwise }\end{cases}
$$

and

$$
\begin{aligned}
\bar{c}\left(\tau: \Phi^{+}\right) & =c\left(1: \tau: \Phi^{+}\right)+c\left(s_{\alpha}: s_{\alpha} \tau: \Phi^{+}\right) \\
& = \begin{cases}2 & \text { if }\langle\alpha, \tau\rangle<0, \\
0 & \text { otherwise. }\end{cases}
\end{aligned}
$$

In this case $\Lambda / \Lambda_{0}=\{0\}$ so that Theorem 2 just says $c\left(w: w^{-1} \tau: \Phi^{+}\right)=\frac{1}{2} \bar{c}\left(\tau: \Phi^{+}\right)$.

Case II. Suppose $\Phi^{+}=\{\alpha, \beta, \gamma, \delta\}$ is of type $B_{2}$ where $\delta$ is the compact root and $\alpha=\gamma+\delta, \beta=\gamma-\delta$. In this case $\Lambda / \Lambda_{0}=\left\{0, \gamma+\Lambda_{0}\right\}$ where $\Phi(\gamma)=\{\gamma, \delta\}$ is of type $A_{1} \times A_{1}$ and

$$
\kappa_{\gamma}(w)= \begin{cases}1 & \text { if } w \in W_{K} \cup s_{\gamma} W_{K}=W_{0}, \\ -1 & \text { if } w \in s_{\alpha} W_{K} \cup s_{\beta} W_{K}=s_{\alpha} W_{0} .\end{cases}
$$

If $\tau \in L$ is written as $\tau=n \alpha+m \beta$, then the nonzero values of $c\left(w: w^{-1} \tau: \Phi^{+}\right)$, $\bar{c}\left(\tau: \Phi^{+}\right)$, and $\bar{c}\left(\tau: \Phi^{+}(\gamma)\right)$ are given by the following table. Since $c\left(w: w^{-1} \tau: \Phi^{+}\right)$ is constant on cosets of $W / W_{0}$, only values for $w=1, s_{\alpha}$ are given.

$\begin{array}{cccc} & 0>m>n & 0>n>m & 0>-n>m \\ c\left(1: \tau: \Phi^{+}\right) & 1 & 1 & 2 \\ c\left(s_{\alpha}: s_{\alpha} \tau: \Phi^{+}\right) & 1 & -1 & 0 \\ \bar{c}\left(\tau: \Phi^{+}\right) & 4 & 0 & 4 \\ \bar{c}\left(\tau: \Phi^{+}(\gamma)\right) & 0 & 4 & 4\end{array}$


Clearly in each case $c\left(w: w^{-1} \tau: \Phi^{+}\right)=\frac{1}{4}\left[\bar{c}\left(\tau: \Phi^{+}\right)+\kappa_{\gamma}(w) \bar{c}\left(\tau: \Phi^{+}(\gamma)\right)\right]$.

Case III. Suppose $\Phi^{+}=\left\{\alpha, \beta, \gamma, \alpha^{\prime}, \beta^{\prime}, \gamma^{\prime}\right\}$ is of type $G_{2}$ where $\alpha$ is simple and short, $\beta$ is simple and long, $\alpha+\beta=\gamma$, and $\alpha^{\prime}, \beta^{\prime}, \gamma^{\prime}$ are orthogonal to $\alpha, \beta, \gamma$, respectively. We assume that $\gamma, \gamma^{\prime}$ are the compact roots. Then $\Lambda / \Lambda_{0}=\{0, \alpha, \beta, \gamma\}$ and $W=W_{K} \cup s_{\alpha} W_{K} \cup s_{\beta} W_{K}$. For each $\lambda \in \Lambda / \Lambda_{0}, \Phi^{+}(\lambda)$ and values of $\kappa_{\lambda}(w)$ are given in the following table.

$\begin{array}{lcccc} & 0 & \alpha & \beta & \gamma \\ \Phi^{+}(\lambda) & \Phi^{+} & \left\{\alpha, \alpha^{\prime}\right\} & \left\{\beta, \beta^{\prime}\right\} & \left\{\gamma, \gamma^{\prime}\right\} \\ \kappa_{\lambda}(1) & 1 & 1 & 1 & 1 \\ \kappa_{\lambda}\left(s_{\alpha}\right) & 1 & 1 & -1 & -1 \\ \kappa_{\lambda}\left(s_{\beta}\right) & 1 & -1 & 1 & -1\end{array}$

For fixed $\tau \in L$, write $\tau=n \alpha+m \alpha^{\prime}$. Then the nonzero values of the $c\left(w: w^{-1} \tau: \Phi^{+}\right)$ and $\bar{c}\left(\tau: \Phi^{+}(\lambda)\right)$ are as follows, verifying the formula of Theorem 2 .

$\begin{array}{lccccc} & 0>3 m>n & m>n>3 m & m<n<0 & 0<n<-m & -m<n<-3 m \\ c\left(1: \tau: \Phi^{+}\right) & 2 & 2 & 4 & 2 & 2 \\ c\left(s_{\alpha}: s_{\alpha} \tau: \Phi^{+}\right) & 2 & 0 & 0 & -2 & 0 \\ c\left(s_{\beta}: s_{\beta} \tau: \Phi^{+}\right) & 0 & -2 & 0 & 0 & 2 \\ \bar{c}\left(\tau: \Phi^{+}\right) & 4 & 0 & 4 & 0 & 4 \\ \bar{c}\left(\tau: \Phi^{+}(\alpha)\right) & 4 & 4 & 4 & 0 & 0 \\ \bar{c}\left(\tau: \Phi^{+}(\beta)\right) & 0 & 0 & 4 & 4 & 4 \\ \bar{c}\left(\tau: \Phi^{+}(\gamma)\right) & 0 & 4 & 4 & 4 & 0\end{array}$

Proof. We first assume that $\Phi^{+}$has been chosen as in (3.3). Write $\varepsilon\left(\lambda: \Phi^{+}\right)=$ $\varepsilon(\lambda)$. For $\tau \in L^{\prime}$, Theorem 2 will be proved by induction on $n$, the rank of $\Phi$. When $n=1,2$, it has been verified above that Theorem 2 holds. We assume that $n \geqslant 3$. The induction step will make use of an identity of Harish-Chandra [3a] which relates the constants $c\left(w: \tau: \Phi^{+}\right)$to constants for root systems of rank $n-1$ as follows.

Let $\alpha \in S$, the set of simple roots for $\Phi^{+}$. Let $\Phi_{\alpha}=\{\beta \in \Phi \mid\langle\beta, \alpha\rangle=0\}$ and $\Phi_{\alpha}^{+}=\Phi^{+} \cap \Phi_{\alpha}$. It is easy to check in each case that $\Phi_{\alpha}^{+}$is a set of positive roots for $\Phi_{\alpha}$ satisfying (3.3). For $\tau \in L^{\prime}$, let $\tau_{\alpha}$ be the unique element in the weight lattice $L_{\alpha}$ of $\Phi_{\alpha}$ satisfying $\left\langle\tau_{\alpha}, \beta\right\rangle=\langle\tau, \beta\rangle$ for all $\beta \in \Phi_{\alpha}$. The Weyl group $W_{\alpha}$ of $\Phi_{\alpha}$ can be considered as a subgroup of $W$.

For $w \in W$ and $\tau \in L$, define

$$
c\left(w: w^{-1} \tau: \Phi_{\alpha}^{+}\right)= \begin{cases}c\left(w_{1}: w_{1}^{-1} \tau_{\alpha}: \Phi_{\alpha}^{+}\right) & \text {if } w=w_{1} w_{2}, w_{1} \in W_{\alpha}, w_{2} \in W_{K}, \\ 0 & \text { if } w \notin W_{\alpha} W_{K} .\end{cases}
$$

Let $s_{\alpha} \in W$ be the reflection corresponding to $\alpha$. Then for $\tau \in L^{\prime}$ :

$$
\begin{gathered}
c\left(w: \tau: \Phi^{+}\right)+c\left(s_{\alpha} w: \tau: \Phi^{+}\right)=c\left(w: \tau: \Phi_{\alpha}^{+}\right)+c\left(s_{\alpha} w: \tau: \Phi_{\alpha}^{+}\right) \\
\bar{c}\left(\tau: \Phi^{+}\right)+\bar{c}\left(s_{\alpha} \tau: \Phi^{+}\right)=2 \bar{c}\left(\tau_{\alpha}: \Phi_{\alpha}^{+}\right) .
\end{gathered}
$$

Identity (3.5) together with the fact that $c\left(w: w^{-1} \tau: \Phi^{+}\right)=0$ if $\tau \in L^{+}=\{\tau \in L \mid$ $\langle\tau, \alpha\rangle>0$ for all $\left.\alpha \in \Phi^{+}\right\}$determines the constants $c\left(w: \tau: \Phi^{+}\right)$inductively [5a]. Thus if we define

$$
s\left(w: \tau: \Phi^{+}\right)=2^{-n} \sum_{\lambda \in \Lambda / \Lambda_{0}} \varepsilon(\lambda) \kappa_{\lambda}(w) \bar{c}\left(w \tau: \Phi^{+}(\lambda)\right),
$$


to prove Theorem 2 for $\tau \in L^{\prime}$ it is only necessary to verify that $s\left(w: w^{-1} \tau: \Phi^{+}\right)=0$ for $\tau \in L^{+}$and that for all $\alpha \in S$,

$$
s\left(w: w^{-1} \tau: \Phi^{+}\right)+s\left(s_{\alpha} w: w^{-1} \tau: \Phi^{+}\right)=c\left(w: w^{-1} \tau: \Phi_{\alpha}^{+}\right)+c\left(s_{\alpha} w: w^{-1} \tau: \Phi_{\alpha}^{+}\right) .
$$

The first identity follows from the fact that for $\tau \in L^{+}$and any $\lambda \in \Lambda,\langle\tau, \alpha\rangle>0$ for all $\alpha \in \Phi^{+}(\lambda) \subseteq \Phi^{+}$so that $\bar{c}\left(\tau: \Phi^{+}(\lambda)\right)=0$. The second identity requires a detailed analysis of the terms occurring in (3.7).

Fix $\tau \in L^{\prime}, w \in W$, and a simple root $\alpha$ and denote the left-hand-side of (3.7) by LHS. Then

$$
\text { LHS }=2^{-n} \sum_{\lambda \in \Lambda / \Lambda_{0}} \varepsilon(\lambda)\left[\kappa_{\lambda}(w) \bar{c}\left(\tau: \Phi^{+}(\lambda)\right)+\kappa_{\lambda}\left(s_{\alpha} w\right) \bar{c}\left(s_{\alpha} \tau: \Phi^{+}(\lambda)\right)\right] .
$$

If $\alpha \notin \Phi(\lambda)$, then since $\alpha$ is simple, $s_{\alpha} \in W\left(\lambda: \Phi^{+}\right)$. Using (3.4),

$$
\begin{aligned}
\varepsilon\left(s_{\alpha} \lambda\right)\left[\kappa_{s_{\alpha} \lambda}(w) \bar{c}(\tau:\right. & \left.\left.\Phi^{+}\left(s_{\alpha} \lambda\right)\right)+\kappa_{s_{\alpha} \lambda}\left(s_{\alpha} w\right) \bar{c}\left(s_{\alpha} \tau: \Phi^{+}\left(s_{\alpha} \lambda\right)\right)\right] \\
& =-\varepsilon(\lambda)\left[\kappa_{\lambda}\left(s_{\alpha} w\right) \bar{c}\left(s_{\alpha} \tau: \Phi^{+}(\lambda)\right)+\kappa_{\lambda}(w) \bar{c}\left(\tau: \Phi^{+}(\lambda)\right)\right],
\end{aligned}
$$

so that if $s_{\alpha} \lambda+\Lambda_{0} \neq \lambda+\Lambda_{0}$, the corresponding terms in LHS cancel, and if $s_{\alpha} \lambda+\Lambda_{0}=\lambda+\Lambda_{0}$, the corresponding term is zero.

Define $\Lambda(\alpha)=\{\lambda \in \Lambda \mid \alpha \in \Phi(\lambda)\}$. If $\lambda \in \Lambda(\alpha)$, then by (3.1), $\kappa_{\lambda}\left(s_{\alpha} w\right)=\kappa_{\lambda}(w)$ and $\alpha$ is a simple root of $\Phi^{+}(\lambda)$ so that using (3.6),

$$
\text { LHS }=2^{-n} \sum_{\lambda \in \Lambda(\alpha) / \Lambda_{0}} \varepsilon(\lambda) \kappa_{\lambda}(w) 2 \bar{c}\left(\tau: \Phi^{+}(\lambda)_{\alpha}\right) .
$$

For any $\lambda \in \Lambda(\alpha), \lambda_{\alpha}=\lambda-\langle\lambda, \alpha\rangle \alpha /\langle\alpha, \alpha\rangle \in\{\lambda \in \Lambda \mid\langle\lambda, \alpha\rangle=0\}$ which can be identified with $\Lambda\left(\Phi_{\alpha}\right)$, the root lattice of $\Phi_{\alpha}$. The mapping $\lambda+\Lambda_{0} \rightarrow \lambda_{\alpha}+\Lambda_{0}\left(\Phi_{\alpha}\right)$ gives a homomorphism of $\Lambda(\alpha) / \Lambda_{0}$ onto $\Lambda\left(\Phi_{\alpha}\right) / \Lambda_{0}\left(\Phi_{\alpha}\right)$ which is bijective if $\alpha \in \Lambda_{0}$ and has kernel $\left\{\Lambda_{0}, \alpha+\Lambda_{0}\right\}$ if $\alpha \notin \Lambda_{0}$. It can be checked case by case (only $\Phi=C_{n}$ or $F_{4}$ are nontrivial) that for $\lambda \in \Lambda(\alpha), \varepsilon(\lambda)=\varepsilon(\lambda+\alpha)$, and that these signs agree with the sign $\varepsilon\left(\lambda_{\alpha}\right)=\varepsilon\left(\lambda_{\alpha}: \Phi_{\alpha}^{+}\right)$. Finally, note $\Phi(\lambda)_{\alpha}=\Phi(\lambda+\alpha)_{\alpha}=\Phi_{\alpha}\left(\lambda_{\alpha}\right)$ and that $\kappa_{\lambda+\alpha}(w)=\kappa_{\lambda}(w)$ if and only if $w^{-1} \alpha \equiv \alpha$.

Case I. Suppose $\alpha \in \Lambda_{0}$. (Since $\operatorname{rank} \Phi \geqslant 3$, this occurs only when $\Phi=C_{n}$ and $\alpha$ is the long simple root.) Then

$$
\text { LHS }=2^{-n+1} \sum_{\lambda \in \Lambda\left(\Phi_{\alpha}\right) / \Lambda_{0}\left(\Phi_{\alpha}\right)} \varepsilon(\lambda) \kappa_{\lambda}(w) \bar{c}\left(\tau: \Phi_{\alpha}^{+}(\lambda)\right) .
$$

If $w \in W_{\alpha} W_{K}$, then LHS is equal to $c\left(w: w^{-1} \tau: \Phi_{\alpha}^{+}\right)$by the induction hypothesis and $s_{\alpha} w \notin W_{\alpha} W_{K}$ so that $c\left(s_{\alpha} w: w^{-1} \tau: \Phi_{\alpha}^{+}\right)=0$. Conversely, if $w \notin W_{\alpha} W_{K}$, then $c\left(w: w^{-1} \tau: \Phi_{\alpha}^{+}\right)=0$, but $s_{\alpha} w \in W_{\alpha} W_{K}$ and LHS $=c\left(s_{\alpha} w: w^{-1} \tau: \Phi_{\alpha}^{+}\right)$.

Case II. Suppose $\alpha \notin \Lambda_{0}$. Then

$$
\begin{aligned}
\text { LHS } & =2^{-n+1} \sum_{\lambda \in \Lambda\left(\Phi_{\alpha}\right) / \Lambda_{0}\left(\Phi_{\alpha}\right)} \varepsilon(\lambda) \bar{c}\left(\tau: \Phi_{\alpha}^{+}(\lambda)\right)\left(\kappa_{\lambda}(w)+\kappa_{\lambda+\alpha}(w)\right) \\
& = \begin{cases}0 & \text { if } w^{-1} \alpha \neq \alpha, \\
2 c\left(w: w^{-1} \tau: \Phi_{\alpha}^{+}\right) & \text {if } w^{-1} \alpha \equiv \alpha .\end{cases}
\end{aligned}
$$


In this case, $w \in W_{\alpha} W_{K}$ if and only if $s_{\alpha} w \in W_{\alpha} W_{K}$ and $c\left(w: w^{-1} \tau: \Phi_{\alpha}^{+}\right)=$ $c\left(s_{\alpha} w: w^{-1} \tau: \Phi_{\alpha}^{+}\right)$. Further, if $w^{-1} \alpha \neq \alpha$, then $w \notin W_{\alpha} W_{K}$ so that $c\left(w: w^{-1} \tau: \Phi_{\alpha}^{+}\right)=$ 0 .

This concludes the proof of Theorem 2 for $\tau \in L^{\prime}$.

Let $\mathcal{F}$ denote the real vector space spanned by $\Phi$. Let $\mathcal{F}^{+}$be any component of $\mathscr{F}^{\prime}=\left\{\tau \in \mathscr{F} \mid \Pi_{\alpha \in \Phi}\langle\tau, \alpha\rangle \neq 0\right\}$. Define $c\left(w: \mathscr{F}^{+}: \Phi^{+}\right)=c\left(w: \tau: \Phi^{+}\right)$where $\tau \in$ $\mathscr{F}^{+} \cap L^{\prime}$. This is independent of the choice of $\tau$ [3a]. Let $\tau \in L^{s}=L \backslash L^{\prime}$. Let $W(\tau)=\{w \in W \mid w \tau=\tau\}$. Let $\mathscr{F}^{+}$be a component of $\mathscr{F}^{\prime}$ with $\tau \in \operatorname{cl}\left(\mathscr{F}^{+}\right)$. Then it follows from the definitions of $c\left(w: \tau: \Phi^{+}\right)$and $\bar{c}\left(\tau: \Phi^{+}\right)$that

$$
c\left(w: \tau: \Phi^{+}\right)=\frac{1}{[W(\tau)]} \sum_{v \in W(\tau)} c\left(w: v \mathcal{F}^{+}: \Phi^{+}\right)
$$

and

$$
\bar{c}\left(\tau: \Phi^{+}\right)=[W(\tau)]^{-1} \sum_{v \in W(\tau)} \bar{c}\left(v \mathscr{F}^{+}: \Phi^{+}\right)
$$

For $\lambda \in \Lambda$, let $\mathscr{F}^{\prime}(\lambda)=\left\{\tau \in \mathscr{F} \mid \prod_{\alpha \in \Phi(\lambda)}\langle\tau, \alpha\rangle \neq 0\right\}$. Let $\mathscr{F}^{+}(\lambda)$ be the component of $\mathscr{F}^{\prime}(\lambda)$ containing $\mathscr{F}^{+}$. Define $U=U(\lambda, \tau)=\left\{w \in W(\tau) \mid w \mathscr{F}^{+} \subseteq \mathscr{F}^{+}(\lambda)\right\}$, and $V=V(\lambda, \tau)=\{w \in W(\Phi(\lambda)) \mid w \tau=\tau\}$. Then $W(\tau)=V U$ and $U \cap V=\{1\}$. For $w \in U$ and $\tau^{\prime} \in \mathscr{F}^{+} \cap L, \bar{c}\left(w \tau^{\prime}: \Phi^{+}(\lambda)\right)=\bar{c}\left(\mathscr{F}^{+}(\lambda): \Phi^{+}(\lambda)\right)$. Thus for $\tau \in L^{s}$, $\tau^{\prime} \in \mathscr{F}^{+} \cap L^{\prime}$, using Theorem 2 for $\tau^{\prime} \in L^{\prime}$,

$$
\begin{aligned}
c\left(w: w^{-1} \tau: \Phi^{+}\right) & =2^{-n} \sum_{\lambda \in \Lambda / \Lambda_{0}} \varepsilon(\lambda) \kappa_{\lambda}(w)[W(\tau)]^{-1} \sum_{v \in W(\tau)} \bar{c}\left(v \tau^{\prime}: \Phi^{+}(\lambda)\right) \\
& =2^{-n} \sum_{\lambda \in \Lambda / \Lambda_{0}} \varepsilon(\lambda) \kappa_{\lambda}(w)[U][W(\tau)]^{-1} \sum_{v \in V} \bar{c}\left(v \mathcal{F}^{+}(\lambda): \Phi^{+}(\lambda)\right) \\
& =2^{-n} \sum_{\lambda \in \Lambda / \Lambda_{0}} \varepsilon(\lambda) \kappa_{\lambda}(w) \bar{c}\left(\tau: \Phi^{+}(\lambda)\right) .
\end{aligned}
$$

This finishes the proof of Theorem 2 for $\Phi^{+}$chosen as in (3.3). Let $v \in W$. Then using (3.2) and the definition of $\varepsilon\left(\lambda: v \Phi^{+}\right)$,

$$
\begin{aligned}
c\left(w: \tau: v \Phi^{+}\right) & =c\left(v^{-1} w: \tau: \Phi^{+}\right) \\
& =2^{-n} \sum_{\lambda \in \Lambda / \Lambda_{0}} \varepsilon\left(\lambda: \Phi^{+}\right) \kappa_{\lambda}\left(v^{-1} w\right) \bar{c}\left(v^{-1} w \tau: \Phi(\lambda) \cap \Phi^{+}\right) \\
& =2^{-n} \sum_{\lambda \in \Lambda / \Lambda_{0}} \varepsilon\left(v^{-1} \lambda: \Phi^{+}\right) \kappa_{\lambda}(v) \kappa_{\lambda}(w) \bar{c}\left(v^{-1} w \tau: \Phi\left(v^{-1} \lambda\right) \cap \Phi^{+}\right) \\
& =2^{-n} \sum_{\lambda \in \Lambda / \Lambda_{0}} \varepsilon\left(\lambda: v \Phi^{+}\right) \kappa_{\lambda}(w) \bar{c}\left(w \tau: \Phi(\lambda) \cap v \Phi^{+}\right) .
\end{aligned}
$$

4. Proof of Theorem 1. Let $H \in \operatorname{Car}(G)$. We will use the notation established in §2. Let $\operatorname{Car}^{\prime}(M)=\operatorname{Car}(M) \backslash\left\{H_{K}\right\}$. Fix $J \in \operatorname{Car}(M)$ with Lie algebra $\dot{\mathrm{i}}=\dot{\mathrm{i}}_{k}+\mathrm{i}_{p}$. Let $W$ be the Weyl group of $\Phi=\Phi\left(\mathrm{m}_{\mathbf{C}}, \dot{\mathrm{l}}_{\mathbf{C}}\right), W_{R}$ and $W_{I}$ the subgroups corresponding to $\Phi_{R}$ and $\Phi_{I}$. Let $W_{\sigma}=\{w \in W \mid w \mathrm{i}=\mathrm{i}\}$. Then $W_{R}, W_{I}$, and $W(M, J)$ are subgroups of $W_{\sigma}$, and as in [5d], we define

$$
w(\mathrm{~m}, \mathrm{i})=\left[W_{R}\right]\left[W\left(M_{J}, J_{K}\right)\right] /[W(M, J)]=\left[W_{R}\right]\left[W_{I}\right] /\left[W_{\sigma}\right] .
$$


Let $\Gamma\left(\dot{i}_{p}\right)$ be a full set of representatives for $W_{R}$ orbits in $Z\left(\dot{i}_{p}\right)$. The representatives can be chosen so that $\Gamma\left(\dot{\mathrm{d}}_{p}\right) \subseteq H_{K}^{0}$. For $\gamma \in \Gamma\left(\dot{\mathrm{d}}_{p}\right)$, let

$$
\Phi_{R}(\gamma)=\left\{\alpha \in \Phi_{R} \mid \xi_{\alpha}(\gamma)=1\right\}, \quad \Phi_{R}^{+}(\gamma)=\Phi_{R}(\gamma) \cap \nu \Phi^{+}\left(g_{\mathbf{C}}, \mathfrak{h}_{\mathbf{C}}\right) \text {. }
$$

Let $W_{R}(\gamma)=W\left(\Phi_{R}(\gamma)\right)$ and let $W_{K}(\gamma)$ be the subgroup of $W_{R}(\gamma)$ generated by reflections in the roots $\alpha$ of $\Phi_{R}(\gamma)$ for which $\nu^{-1} \alpha$ is a compact root of $\left(m, \mathfrak{h}_{k}\right)$. Let $\mathrm{i}_{p}^{+}(\gamma)=\left\{H \in \dot{\mathrm{i}}_{p} \mid \alpha(H)>0\right.$ for all $\left.\alpha \in \Phi_{R}^{+}(\gamma)\right\}$. Then for $\gamma \in \Gamma\left(\dot{\mathrm{i}}_{p}\right), h \in \exp \left(i \mathrm{i}_{p}\right)$, and $\mu \in i_{p}^{*}$, we define

$$
\begin{aligned}
I_{J}^{H}(\gamma: h: \mu)= & {\left[W_{K}(\gamma)\right]^{-1} \sum_{v \in W_{R}(\gamma)} \operatorname{det} v \int_{\mathrm{i}_{p}^{+}(\gamma)} \sum_{\tau \in L\left(\Phi_{R}\right)} \overline{\xi_{\tau}(\gamma v h)} } \\
& \times c\left(v: v^{-1} \tau: \Phi_{R}^{+}(\gamma)\right) \exp (\tau-i \mu)(H) d H .
\end{aligned}
$$

Let $\delta(H, J)$ be the set of all sequences $J_{0}, J_{1}, \ldots, J_{l}$ where $J_{0}=H, \tilde{J}_{l}=\tilde{J}=J H_{p}$, and for $1 \leqslant i \leqslant l, J_{i} \in \operatorname{Car}^{\prime}\left(M_{\tilde{J}_{i-1}}\right)$. For $0 \leqslant i \leqslant l$, let $\mathrm{a}_{i}=\left(\dot{\mathrm{d}}_{i}\right)_{p}$. Then $\dot{\mathrm{i}}_{p}=\bigoplus \sum_{i=1}^{l} \mathrm{a}_{i}$. For $h_{k} \in H_{K}$, write $\nu\left(h_{k}\right)=h_{0} h_{1} \cdots h_{l}$ where $h_{0} \in J_{K}$ and for $1 \leqslant i \leqslant l, h_{i} \in$ $\exp \left(i a_{i}\right)$. For $\mu \in j_{p}^{*}$, write $\mu_{i}$ for the restriction of $\mu$ to $a_{i}$.

THEOREM 4.3. There is a dense open set $H^{*} \subseteq H^{\prime}$ so that for $h=h_{k} h_{p} \in H^{*}$, $f \in C_{c}^{\infty}(G)$,

$$
\begin{aligned}
F_{f}^{H}(h)= & \frac{(-1)^{r_{I}(H)}\left[W\left(M, H_{K}\right): W\left(M^{0}, H_{K}^{0}\right)\right]}{\operatorname{vol}\left(H_{K}\right)(2 \pi)^{\operatorname{dimh} \mathfrak{h}_{p}}} \sum_{w \in W\left(M^{0}, H_{K}^{0}\right)} \operatorname{det} w \\
& \times \sum_{J \in \operatorname{Car}(M)}(4 \pi)^{-\operatorname{dimi}_{p}}\left[W\left(M_{J}, J_{K}\right)\right]^{-1} \sum_{b^{*} \in \hat{J}_{K}} \int_{\mathfrak{h}_{p}^{*}} h_{p}^{-i \mu^{\prime}} \int_{\mathfrak{i}_{p}^{*}} \theta\left(\tilde{J}, b^{*}, \mu^{\prime} \otimes \mu\right)(f) \\
& \times \sum_{S \in \delta(H, J)}(-1)^{l} I\left(S: b^{*}: \mu: w h_{k}\right) d \mu d \mu^{\prime} .
\end{aligned}
$$

For $S \in S(H, J), b^{*} \in \hat{J}_{K}, \mu \in \mathrm{i}_{p}^{*}$, and $\nu h_{k}=h_{0} h_{1} \cdots h_{l} \in H_{K}$,

$$
I\left(S: b^{*}: \mu: h_{k}\right)=\sum_{\gamma_{1} \in \Gamma\left(a_{1}\right)} \cdots \sum_{\gamma_{l} \in \Gamma\left(a_{l}\right)} \overline{b^{*}\left(\gamma_{1} \cdots \gamma_{l} h_{0}\right)} \prod_{i=1}^{l} w_{i} I_{J_{i}}^{\tilde{J_{i-1}}}\left(\gamma_{i}: h_{i}: \mu_{i}\right) .
$$

Here $w_{i}=w\left(\mathrm{~m}_{i-1}, \dot{\mathrm{l}}_{i}\right) /\left[Z\left(\mathrm{a}_{i}\right) \cap Z\left(\tilde{\mathrm{a}}_{i-1}\right)\right]$ where $\tilde{\mathfrak{a}}_{i}=\oplus \sum_{j=0}^{i} \mathfrak{a}_{j}, 0 \leqslant i \leqslant l$.

Proof. This result is proved in the same way as Theorem 3.7 of [5d], using Theorem 3.4 of [5d]. Note that for $J \in \operatorname{Car}^{\prime}(M), W\left(M_{J}^{0}, J_{K}^{0}\right) \subseteq \nu\left(W\left(M^{0}, H_{K}^{0}\right)\right)$, so that all Weyl group sums can be combined. The subset $H^{*}$ of $H^{\prime}$ for which the formula is valid will be smaller than that for Theorem 3.4 [5d], as when each remainder term is analyzed, it is necessary to eliminate certain elements.

The remainder of this section will be devoted to evaluating the integrals $I_{J}^{H}$, $H \in \operatorname{Car}(G), J \in \operatorname{Car}^{\prime}\left(M_{H}\right)$, which occur in the inversion formula. Sally and Warner derived a simple formula for $I_{J}^{H}(\gamma: h: \mu)$ in [10] when $\Phi$ is of type $A_{1}$. For rank $\Phi \geqslant 2$. Theorem 2 will be used to express $I_{J}^{H}$ in terms of factors of the type which occur in the Fourier inversion of stable orbital integrals, which are then simplified as in [5d, e] into products of rank one and two type factors. 
Fix $\gamma \in \Gamma\left(\dot{\mathrm{i}}_{p}\right)$. Write $\Phi=\Phi_{R}(\gamma), \Phi_{C P T}=\left\{\alpha \in \Phi \mid \nu^{-1} \alpha\right.$ is a compact root of $\left.\left(\mathfrak{m}, \mathfrak{h}_{k}\right)\right\}, \mathfrak{a}^{+}=\mathrm{i}_{p}^{+}(\gamma), W=W_{R}(\gamma)=W(\Phi)$, and $W_{K}=W\left(\Phi_{C P T}\right)$. We will use the notation of $\S \S 2$ and 3 . Let $\Lambda_{1}$ be a subset of $\Lambda$ so that for each $\lambda \in \Lambda$ there is a unique $\lambda_{1} \in \Lambda_{1}$ with $\lambda \in w \lambda_{1}+\Lambda_{0}$ for some $w \in W$. Then every coset $\lambda+\Lambda_{0}$ can be represented by one of the form $\sigma \lambda+\Lambda_{0}$ where $\lambda \in \Lambda_{1}$ and $\sigma \in W\left(\lambda: \Phi^{+}\right)=\{\sigma$ $\left.\in W \mid \sigma \Phi^{+}(\lambda) \subseteq \Phi^{+}\right\}$. This representation is unique only up to an element of $W_{1}\left(\Phi: \Phi^{+}(\lambda)\right)=\left\{\sigma \in W \mid \sigma \Phi^{+}(\lambda)=\Phi^{+}(\lambda)\right\}$. Fix $\lambda \in \Lambda_{1}$ and $\varphi=\varphi(\lambda) \in$ $\widetilde{T}(\Phi(\lambda))$. Write $\varphi=\varphi_{1} \cup \cdots \cup \varphi_{s}$ where the $\varphi_{i}$ are simple. Let $a_{i}=\Sigma_{\alpha \in \varphi_{i}} \mathbf{R} H_{\alpha}$, $1 \leqslant i \leqslant s$. Then $\dot{i}_{p}=a_{1} \oplus \cdots \oplus a_{s}$. Write $T_{0}=\exp \left(i \dot{\mathrm{j}}_{p}\right)$ and $T_{j}=\exp \left(i \mathrm{a}_{j}\right), 1 \leqslant j \leqslant$ s. Then $T_{0}=T_{1} \cdots T_{s}$, but the product need not be direct. Thus for $\tau \in L(\varphi), \xi_{\tau}$ need not be well defined on $T_{0}$, although it is on $\tilde{T}_{0}=T_{1} \times \cdots \times T_{s}$, the abstract direct product, by $\xi_{\tau}\left(\eta_{1}, \ldots, \eta_{s}\right)=\Pi_{j=1}^{s} \xi_{\tau_{j}}\left(\eta_{j}\right)$ where $\tau_{j}$ is the restriction of $\tau$ to $a_{j}$. Let $E=\left\{\left(\eta_{1}, \ldots, \eta_{s}\right) \in \tilde{T}_{0} \mid \Pi_{j=1}^{s} \eta_{i}=1\right\}$. Write $L_{R}=L\left(\Phi_{R}(\mathrm{~m}, \dot{\mathrm{l}})\right)$. Then $[E]=$ $\left[L(\varphi): L_{R}\right]$, and for $\tau \in L(\varphi)$,

$$
\sum_{\left(\eta_{1}, \ldots, \eta_{s}\right) \in E} \xi_{\tau}\left(\eta_{1}, \ldots, \eta_{s}\right)= \begin{cases}{[E]} & \text { if } \tau \in L_{R}, \\ 0 & \text { if } \tau \notin L_{R} .\end{cases}
$$

For $\mu \in \mathrm{i}_{p}^{*}$, let $\mu_{j}$ denote the restriction of $\mu$ to $\mathfrak{a}_{j}, 1 \leqslant j \leqslant s$. For $h \in T_{0}$, write $h=\prod_{j=1}^{s} h_{j}$ where $h_{j} \in T_{j}$. This decomposition is unique only up to componentwise multiplication by an element of $E$. Define

$$
P\left(\varphi^{+}, \mu, h\right)=\sum_{\left(\eta_{1}, \ldots, \eta_{s}\right) \in E} \prod_{i=1}^{s} S\left(\varphi_{i}^{+}, \mu_{i}, \eta_{i} h_{i}\right)
$$

where the $S\left(\varphi_{i}^{+}, \mu_{i}, h_{i}\right)$ are defined as in Theorem 1. Define

$$
c(\lambda)=\varepsilon\left(\varphi: \Phi^{+}(\lambda)\right) \varepsilon\left(\lambda: \Phi^{+}\right) 2^{n}[E]\left[W_{1}\left(\Phi: \Phi^{+}(\lambda)\right)\right]\left[W_{1}\left(\Phi(\lambda): \varphi^{+}\right)\right]
$$

where $n=\operatorname{rank} \Phi$, and for any $\operatorname{root}$ system $\Phi$ and subset $\psi, W_{1}(\Phi: \psi)=\{w \in$ $W(\Phi) \mid w \psi=\psi\}$.

To shorten formulas, we use the following notation. For $b^{*} \in \hat{J}_{K}, \mu \in \mathrm{i}_{p}^{*}, h_{k} \in H_{K}^{\prime}$, we say $g\left(b^{*}, \mu, h_{k}\right) \equiv g^{\prime}\left(b^{*}, \mu, h_{k}\right)$ if for $h_{p} \in H_{p}^{\prime}$,

$$
\begin{aligned}
\sum_{b^{*} \in \hat{J}_{K}} \int_{\mathfrak{h}_{p}^{*}} h_{p}^{-i \mu^{\prime}} \int_{\mathrm{i}_{p}^{*}} \theta\left(\tilde{J}, b^{*}, \mu \otimes \mu^{\prime}\right)(f) g\left(b^{*}, \mu, h_{k}\right) d \mu d \mu^{\prime} \\
=\sum_{b^{*} \in \hat{J}_{K}} \int_{\mathfrak{h}_{p}^{*}} h_{p}^{-i \mu^{\prime}} \int_{\mathrm{i}_{p}^{*}} \theta\left(\tilde{J}, b^{*}, \mu \otimes \mu^{\prime}\right)(f) g^{\prime}\left(b^{*}, \mu, h_{k}\right) d \mu d \mu^{\prime},
\end{aligned}
$$

where $g$ and $g^{\prime}$ are functions of $\left(b^{*}, \mu, h_{k}\right)$ for which the sums and integrals above converge. Because of the transformation rule for $\theta\left(\tilde{J}, b^{*}, \mu \otimes \mu^{\prime}\right)(f)$, for $w \in$ $W_{R}(\mathfrak{m}, \mathrm{i})$,

$$
g\left(b^{*}, \mu, h_{k}\right) \equiv g\left(\eta(w) \otimes w b^{*}, w \mu, h_{k}\right)
$$

where $\eta(w) \in \hat{J}_{K}$ is defined as in [5d]. Recall that $\left(\eta(w) \otimes w b^{*}\right)\left(j_{k}\right)=b^{*}\left(j_{k}\right)$ if $w j_{k}=j_{k}, j_{k} \in J_{K}$. 
LEMma 4.7 For $h_{K} \in H_{K}$, decompose $\nu h_{K}=h_{0} h_{1}$ where $h_{0} \in J_{K}$ and $h_{1} \in \exp \left(i \dot{1}_{p}\right)$. Then

$$
\begin{aligned}
\overline{b^{*}\left(\gamma h_{0}\right)} I_{J}^{H}\left(\gamma: h_{1}: \mu\right) \equiv & (\pi i)^{n}\left[W_{K}\right]^{-1} \overline{b^{*}\left(\gamma h_{0}\right)} \\
& \times \sum_{\lambda \in \Lambda_{1}} c(\lambda)^{-1} \sum_{v \in W} \operatorname{det} v \kappa_{\lambda}(v) P\left(\varphi^{+}(\lambda), \mu, \gamma v h_{1}\right) .
\end{aligned}
$$

Proof. Using (4.2) and Theorem 2,

$$
\overline{b^{*}\left(\gamma h_{0}\right)} I_{J}^{H}\left(\gamma: h_{1}: \mu\right) \equiv\left[W_{K}\right]^{-1} 2^{-n} \overline{b^{*}\left(\gamma h_{0}\right)} \sum_{\lambda \in \Lambda_{1}} I\left(\lambda, \mu, \gamma h_{1}\right)
$$

where for $\lambda \in \Lambda_{1}$ and $j \in \exp \left(i i_{p}\right)$,

$$
\begin{aligned}
I(\lambda, \mu, j) & =\left[W_{1}\left(\Phi: \Phi^{+}(\lambda)\right)\right]^{-1} \sum_{\sigma \in W\left(\lambda: \Phi^{+}\right)} \sum_{v \in W} \operatorname{det} v \\
& \times \int_{a^{+}} \sum_{\tau \in L_{R}} \overline{\xi_{\tau}(v j)} \varepsilon\left(\sigma \lambda: \Phi^{+}\right) \kappa_{\sigma \lambda}(v) \bar{c}\left(\tau: \sigma \Phi^{+}(\lambda)\right) \exp (\tau-i \mu)(H) d H .
\end{aligned}
$$

Fix $\lambda \in \Lambda_{1}$. Write $W_{0}=W\left(\lambda: \Phi^{+}\right), W_{1}=W_{1}\left(\Phi: \Phi^{+}(\lambda)\right)$, and $\varepsilon\left(\sigma \lambda: \Phi^{+}\right)=$ $\varepsilon(\sigma \lambda)$. Then using (3.4),

$$
\begin{aligned}
I(\lambda, \mu, j)= & {\left[W_{1}\right]^{-1} \sum_{\sigma \in W_{0}} \sum_{v \in W} \operatorname{det}(\sigma v) } \\
& \times \int_{a^{+}} \sum_{\tau \in L_{R}} \overline{\xi_{\sigma \tau}(\sigma v j)} \varepsilon(\sigma \lambda) \kappa_{\sigma_{\lambda}}(\sigma v) \bar{c}\left(\sigma \tau: \sigma \Phi^{+}(\lambda)\right) \exp (\sigma \tau-i \mu)(H) d H \\
= & \varepsilon(\lambda)\left[W_{1}\right]^{-1} \sum_{v \in W} \operatorname{det} v \kappa_{\lambda}(v) \sum_{\sigma \in W_{0}} \\
& \times \int_{\sigma^{-1} a^{+}} \sum_{\tau \in L_{R}} \overline{\xi_{\tau}(v j)} \bar{c}\left(\tau: \Phi^{+}(\lambda)\right) \exp \left(\tau-i \sigma^{-1} \mu\right)(H) d H .
\end{aligned}
$$

Let $\varphi \in \mathcal{T}(\Phi(\lambda))$. Define $W_{0}(\varphi, \lambda)=\left\{s \in W(\Phi(\lambda)) \mid s \varphi^{+} \subseteq \Phi^{+}(\lambda)\right\}$. Then as in $[5 \mathbf{c}, \mathbf{e}] \quad \mathcal{T}(\Phi(\lambda))=\left\{\sigma \varphi \mid \sigma \in W_{0}(\varphi, \lambda)\right\}, \quad \varepsilon\left(\sigma \varphi: \Phi^{+}(\lambda)\right)=\operatorname{det} \sigma \varepsilon\left(\varphi: \Phi^{+}(\lambda)\right)$, and (2.1) can be rewritten as

$$
\bar{c}\left(\tau: \Phi^{+}(\lambda)\right)=\left[W_{1}\left(\Phi(\lambda): \varphi^{+}\right)\right]^{-1} \varepsilon\left(\varphi: \Phi^{+}(\lambda)\right) \sum_{s \in W_{0}(\varphi, \lambda)} \operatorname{det} s \bar{c}\left(s^{-1} \tau: \varphi^{+}\right) .
$$

Write $W_{1}\left(\Phi(\lambda): \varphi^{+}\right)=W_{1}^{\prime}, W_{0}(\varphi, \lambda)=W_{0}^{\prime}$, and $\varepsilon(\varphi)=\varepsilon\left(\varphi: \Phi^{+}(\lambda)\right)$. Thus, using (3.1),

$$
\begin{aligned}
I(\lambda, \mu, j)= & \varepsilon(\lambda)\left[W_{1}\right]^{-1}\left[W_{1}^{\prime}\right]^{-1} \varepsilon(\varphi) \sum_{s \in W_{0}^{\prime}} \operatorname{det} s \sum_{v \in W} \operatorname{det} s v \kappa_{\lambda}(s v) \\
& \times \sum_{\sigma \in W_{0}} \int_{s^{-1} \sigma^{-1} \mathfrak{a}^{+}} \sum_{\tau \in L_{R}} \overline{\xi_{s \tau}(s v j)} \bar{c}\left(\tau: \varphi^{+}\right) \exp \left(\tau-i s^{-1} \sigma^{-1} \mu\right)(H) d H \\
= & \varepsilon(\lambda)\left[W_{1}\right]^{-1}\left[W_{1}^{\prime}\right]^{-1} \varepsilon(\varphi) \sum_{v \in W} \operatorname{det} v \kappa_{\lambda}(v) \\
& \times \sum_{\sigma \in W_{0}} \sum_{s \in W_{0}^{\prime}} I\left(v j, s^{-1} \sigma^{-1} \mu, s^{-1} \sigma^{-1} \mathfrak{a}^{+}\right)
\end{aligned}
$$


where

$$
I\left(j, \mu, \mathfrak{a}^{+}\right)=\int_{\mathfrak{a}^{+}} \sum_{\tau \in L_{R}} \overline{\xi_{\tau}(j)} \bar{c}\left(\tau: \varphi^{+}\right) \exp (\tau-i \mu)(H) d H,
$$

for $j \in \exp \left(i a_{j}\right), \mu \in \mathrm{i}_{p}^{*}$, and $\mathrm{a}^{+}$a component of $\mathfrak{a}^{\prime}=\{H \in \mathfrak{a} \mid \alpha(H) \neq 0$ for all $\alpha \in \Phi\}$.

Because every element of $W=W_{R}(\gamma)$ centralizes $\gamma h_{0}$, using (4.6),

$$
\overline{b^{*}\left(\gamma h_{0}\right)} I\left(\gamma v h_{1}, s^{-1} \sigma^{-1} \mu, s^{-1} \sigma^{-1} \mathfrak{a}^{+}\right) \equiv \overline{b^{*}\left(\gamma h_{0}\right)} I\left(\gamma v h_{1}, \mu, s^{-1} \sigma^{-1} \mathfrak{a}^{+}\right)
$$

for all $v \in W, s \in W_{0}^{\prime}$, and $\sigma \in W_{0}$. Note that

$$
\bigcup_{s \in W_{0}^{\prime}} \bigcup_{\sigma \in W_{0}} s^{-1} \sigma^{-1} a^{+}=\mathfrak{a}^{+}(\varphi)=\left\{H \in \mathrm{i}_{p} \mid \alpha(H)>0 \text { for all } \alpha \in \varphi^{+}\right\} \text {. }
$$

Thus

$$
\begin{aligned}
\overline{b^{*}\left(\gamma h_{0}\right)} I\left(\lambda, \mu, \gamma h_{1}\right) \equiv \varepsilon(\lambda)\left[W_{1}\right]^{-1}\left[W_{1}^{\prime}\right]^{-1} \varepsilon(\varphi) \overline{b^{*}\left(\gamma h_{0}\right)} \\
\quad \times \sum_{v \in W} \operatorname{det} v \kappa_{\lambda}(v) \int_{\mathrm{a}^{+}(\varphi)} \sum_{\tau \in L_{R}} \overline{\xi_{\tau}\left(\gamma v h_{1}\right)} \bar{c}\left(\tau: \varphi^{+}\right) \exp (\tau-i \mu)(H) d H .
\end{aligned}
$$

Using the notation of (4.5), write $\mathfrak{a}_{i}^{+}=\left\{H \in \mathfrak{a}_{i} \mid \alpha(H)>0\right.$ for all $\left.\alpha \in \varphi_{i}^{+}\right\}$and $\gamma v h_{1}=\prod_{i=1}^{s} h_{i}(v)$ where $h_{i}(v) \in T_{i}, 1 \leqslant i \leqslant s$. For any $\mu \in \mathfrak{a}_{i}^{*}, h \in T_{i}$, write

$$
\bar{I}\left(h: \mu: \varphi_{i}^{+}\right)=\int_{\mathfrak{a}_{i}^{+}} \sum_{\tau \in L\left(\varphi_{i}\right)} \overline{\xi_{\tau}(h)} \bar{c}\left(\tau: \varphi_{i}^{+}\right) \exp (\tau-i \mu)(H) d H .
$$

Then using (4.4),

$$
\begin{aligned}
\overline{b^{*}\left(\gamma h_{0}\right)} I\left(\lambda, \mu, \gamma h_{1}\right) \equiv & 2^{n} c(\lambda)^{-1} \overline{b^{*}\left(\gamma h_{0}\right)} \sum_{v \in W} \operatorname{det} v \kappa_{\lambda}(v) \\
& \times \sum_{\left(\eta_{1}, \ldots, \eta_{s}\right) \in E} \prod_{i=1}^{s} \bar{I}\left(\eta_{i} h_{i}(v): \mu_{i}: \varphi_{i}^{+}\right) .
\end{aligned}
$$

The factors $\bar{I}\left(h: \mu: \varphi_{i}^{+}\right)$defined above are exactly the terms associated with the simple root system $\varphi_{i}$ of types $A_{1}$ or $B_{2}$ which arise in the Fourier inversion formulas of stable orbital integrals. It follows from results of Sally and Warner [10] for the $A_{1}$ case and Chao [2] for the $B_{2}$ case, which are summarized in Lemma 4.6 [5d], that

$$
\begin{aligned}
\overline{b^{*}\left(\gamma h_{0}\right)} \sum_{u \in W(\varphi)} \operatorname{det} u \sum_{\left(\eta_{1}, \ldots, \eta_{s}\right) \in E} \prod_{i=1}^{s} \bar{I}\left(\eta_{i} u h_{i}(v): \mu_{i}: \varphi_{i}^{+}\right) \\
\equiv(\pi i)^{n} \overline{b^{*}\left(\gamma h_{0}\right)} \sum_{u \in W(\varphi)} \operatorname{det} u P\left(\varphi^{+}, \mu, u \gamma v h_{1}\right) .
\end{aligned}
$$

The lemma now follows from observing that $u h_{i}(v)=h_{i}(u v)$ for all $u \in W(\varphi)$, $v \in W$, and $1 \leqslant i \leqslant s$, and that $W(\varphi) \subseteq W(\Phi(\lambda))$ so that $\kappa_{\lambda}(u v)=\kappa_{\lambda}(v)$ for all $u \in W(\varphi), v \in W$.

In (4.7) we have obtained an expression for $I_{J}^{H}$ in terms of factors $S\left(\varphi^{+}, \mu, h\right), \varphi$ of types $A_{1}$ or $B_{2}$, which occur in the Fourier inversion formulas for stable orbital integrals. In Lemma 4.9 we will do more simplification to eliminate the sum over $\Lambda_{1}$ and the "characters" $\kappa_{\lambda}$. 
Fix $\psi=\varphi_{1} \cup \cdots \cup \varphi_{s} \in \mathcal{T}(\Phi)$ for which every long root is of noncompact type. (We call $\alpha$ a long root of $\psi$ if $\alpha$ belongs to any factor $\varphi_{i}$ of type $A_{1}$ or if $\alpha$ is a long root of a factor $\varphi_{i}$ of type $B_{2}$.) We use the notation of (4.5). Let $1 \leqslant i \leqslant s$. Let $\mu \in a_{i}^{*}, h \in T_{i}$. Define $T\left(\varphi_{i}^{+}, \mu, h\right)=S\left(\varphi_{i}^{+}, \mu, h\right)$ if $\varphi_{i}$ is of type $A_{1}$. If $\varphi_{i}$ is of type $B_{2}$, let $\varphi_{i, s}^{+}$denote the short positive roots of $\varphi_{i}$, and define $S\left(\varphi_{i, s}^{+}, \mu, h\right)$ as in Theorem 1. Then define $T\left(\varphi_{i}^{+}, \mu, h\right)=S\left(\varphi_{i}^{+}, \mu, h\right)+\frac{1}{4} S\left(\varphi_{i, s}^{+}, \mu, h\right)$. Set

$$
Q\left(\psi^{+}, \mu, h\right)=\sum_{\left(\eta_{1}, \ldots, \eta_{s}\right) \in E} \prod_{i=1}^{s} T\left(\varphi_{i}^{+}, \mu_{i}, \eta_{i}, h_{i}\right) .
$$

LEMMA 4.9. Let $\psi \in \mathcal{T}(\Phi)$ for which every long root of $\psi$ is of noncompact type. Then

$$
\begin{aligned}
& \sum_{w \in W\left(M^{0}, H_{K}^{0}\right)} \operatorname{det} w \overline{b^{*}\left(\gamma h_{0}(w)\right)} I_{J}^{H}\left(\gamma: h_{1}(w): \mu\right) \\
& \equiv(\pi i)^{n}\left[W_{1}\left(\Phi: \psi^{+}\right)\right]^{-1}\left[L_{\psi}: L_{R}\right]^{-1} \varepsilon\left(\psi: \Phi^{+}\right) \\
& \times \sum_{w \in W\left(M^{0}, H_{K}^{0}\right)} \operatorname{det} w \overline{b^{*}\left(\gamma h_{0}(w)\right)} Q\left(\psi^{+}, \mu, \gamma h_{1}(w)\right) .
\end{aligned}
$$

Proof. Suppose first that $\Phi^{+}$has been chosen so that $\Phi^{+}$and $\psi^{+}=\psi \cap \Phi^{+}$both satisfy (3.3). Then $\Lambda_{1}$ can be chosen so that for all $\lambda \in \Lambda_{1}$ :

(i) $\varepsilon\left(\lambda: \Phi^{+}\right)=1$;

(ii) $\varphi(\lambda)=\psi \cap \Phi(\lambda) \in \mathcal{T}(\Phi(\lambda))$ and $\varepsilon\left(\varphi(\lambda): \Phi^{+}(\lambda)\right)=\varepsilon\left(\psi: \Phi^{+}\right)$;

(iii) $W_{1}\left(\Phi: \Phi^{+}(\lambda)\right) \subseteq W_{1}\left(\Phi: \varphi^{+}\right)$;

(iv) $\lambda$ is a sum of long roots of $\Phi(\lambda)$.

Fix $\varphi \subseteq \psi$ such that $\Lambda_{1}(\varphi)=\left\{\lambda \in \Lambda_{1} \mid \varphi \in \mathcal{T}(\Phi(\lambda))\right\} \neq \varnothing$. Let $U(\varphi)=$ $S(\varphi) W_{1}\left(\Phi: \varphi^{+}\right)$where $S(\varphi)$ is the subgroup of $W(\varphi)$ generated by reflections in long roots of $\varphi$. It is clear from the explicit formulas of the $S\left(\varphi_{i}^{+}, \mu_{i}, h_{i}\right)$ in Theorem 1 that for $s \in S(\varphi), P\left(\varphi^{+}, \mu, s h\right)=\operatorname{det} s P\left(\varphi^{+}, \mu, h\right)$ for all $\mu \in \mathrm{i}_{p}^{*}$ and $h \in$ $\exp \left(i \dot{\mathrm{i}}_{p}\right)$. For $u \in W_{1}\left(\Phi: \varphi^{+}\right), P\left(\varphi^{+}, \mu, u h\right)=P\left(\phi^{+}, u^{-1} \mu, h\right)$. Finally, $\nu^{-1} W_{K} \subseteq$ $W\left(M^{0}, H_{K}^{0}\right)$. Thus

$$
\begin{aligned}
& \sum_{\lambda \in \Lambda_{1}(\varphi)} c(\lambda)^{-1}\left[W_{K}\right]^{-1} \sum_{w \in W\left(M^{0}, H_{K}^{0}\right)} \operatorname{det} w \overline{b^{*}\left(\gamma h_{0}(w)\right)} \\
& \times \sum_{v \in W} \operatorname{det} v \kappa_{\lambda}(v) P\left(\varphi^{+}, \mu, \gamma v h_{1}(w)\right) \\
& \equiv {\left[U(\varphi) \cap W_{K}\right]^{-1} \sum_{w \in W\left(M^{0}, H_{K}^{0}\right)} \operatorname{det} w \overline{b^{*}\left(\gamma h_{0}(w)\right)} } \\
& \times \sum_{v \in U(\varphi) \backslash W / W_{K}} \operatorname{det} v P\left(\varphi^{+}, \mu, \gamma v h_{1}(w)\right) \\
& \times[S(\varphi)] \sum_{\lambda \in \Lambda_{1}(\varphi)} c(\lambda)^{-1} \sum_{u \in W_{1}\left(\Phi: \varphi^{+}\right)} \operatorname{det} u \kappa_{\lambda}(u v) .
\end{aligned}
$$

Using (3.2), $\kappa_{\lambda}(u v)=\kappa_{\lambda}(u) \kappa_{u^{-1} \lambda}(v)$. In the case that $\varphi=\psi, \psi \in \mathcal{J}(\Phi)$ implies that det $u=1$ for all $u \in W_{1}\left(\Phi: \psi^{+}\right)$. Since all long roots of $\psi$ are noncompact, and because of assumption (iv) on $\lambda, u^{-1} \lambda \equiv \lambda$ for all $u \in W_{1}\left(\Phi: \psi^{+}\right)$so that $\kappa_{\lambda}(u)=1$. 
In the few cases that $\varphi \subsetneq \psi$ it is easy to check that det $u=\kappa_{\lambda}(u)$ for all $u \in$ $W_{1}\left(\Phi: \varphi^{+}\right)$. Thus det $u \kappa_{\lambda}(u v)=\kappa_{u^{-1} \lambda}(v)$ for all $u \in W_{1}\left(\Phi: \varphi^{+}\right)$.

Clearly $\varphi \in \mathcal{T}(\Phi(u \lambda))$ for all $u \in W_{1}\left(\Phi: \varphi^{+}\right), \lambda \in \Lambda_{1}(\varphi)$. Conversely, if $\varphi \in$ $\mathcal{T}(\Phi(\lambda))$ for any $\lambda \in \Lambda$, then $\lambda+\Lambda_{0}=w \lambda_{1}+\Lambda_{0}$ for a unique $\lambda_{1} \in \Lambda_{1}(\varphi)$. Since $\varphi, w^{-1} \varphi \in \mathcal{T}\left(\Phi\left(\lambda_{1}\right)\right)$, there is $\sigma \in W\left(\Phi\left(\lambda_{1}\right)\right)$ with $w^{-1} \varphi^{+}=\sigma \varphi^{+}$so that $u=w \sigma \in$ $W_{1}\left(\Phi: \varphi^{+}\right)$and $\Phi\left(u \lambda_{1}\right)=\Phi(\lambda)$. Further, for $u_{1}, u_{2} \in W_{1}\left(\Phi: \varphi^{+}\right), \Phi\left(u_{1} \lambda\right)=$ $\Phi\left(u_{2} \lambda\right)$ if and only if $u_{1}^{-1} u_{2} \in\left\{u \in W(\Phi) \mid u \Phi(\lambda)=\Phi(\lambda), u \varphi^{+}=\varphi^{+}\right\}=$ $W_{1}\left(\Phi: \Phi^{+}(\lambda)\right) W_{1}\left(\Phi(\lambda): \varphi^{+}\right)$.

Thus

$$
\begin{aligned}
{[S(\varphi)] \sum_{\lambda \in \Lambda_{1}(\varphi)} c(\lambda)^{-1} } & \sum_{u \in W_{1}\left(\Phi: \varphi^{+}\right)} \operatorname{det} u \kappa_{\lambda}(u v) \\
& =\varepsilon\left(\psi: \Phi^{+}\right)\left[L_{\varphi}: L_{R}\right]^{-1} \sum_{\lambda+\Lambda_{0} \neq \varphi \in \mathcal{T}(\Phi(\lambda))} \kappa_{\lambda}(v) .
\end{aligned}
$$

Case I. Suppose that $\varphi=\psi$. Then $\{\lambda \in \Lambda \mid \psi \in \mathscr{T}(\Phi(\lambda))\}=\{\lambda \in \Lambda \mid \psi \subseteq \Phi(\lambda)\}$ is a sublattice of $\Lambda$ containing $\Lambda_{0}$ which we will denote by $\Lambda_{\psi}$. Further, for fixed $v \in W, \lambda \rightarrow \kappa_{\lambda}(v)$ is a character $\Lambda_{\psi} / \Lambda_{0}$. Thus

$$
\sum_{\lambda \in \Lambda_{\psi} / \Lambda_{0}} \kappa_{\lambda}(v)= \begin{cases}{\left[\Lambda_{\psi}: \Lambda_{0}\right]} & \text { if } \kappa_{\lambda}(v)=1 \text { for all } \lambda \in \Lambda_{\psi}, \\ 0 & \text { otherwise. }\end{cases}
$$

Note $\Lambda_{\psi}=\{\lambda \in \Lambda \mid\langle\lambda, \alpha\rangle /\langle\alpha, \alpha\rangle \in Z$ for all $\alpha \in \psi\}$ and $\Lambda_{0}=\{\lambda \in \Lambda \mid$ $\langle\lambda, \alpha\rangle /\langle\alpha, \alpha\rangle \in Z$ for all $\alpha \in \Phi\}$. Thus $\left[\Lambda_{\psi}: \Lambda_{0}\right]=\left[L_{\psi}: L\right]$ and $[E]^{-1}\left[\Lambda_{\psi}: \Lambda_{0}\right]=$ $\left[L: L_{R}\right]^{-1}$. It can be checked that $\Lambda_{\psi} / \Lambda_{0}=\left\{\sum_{i=1}^{k} \alpha_{i}+\Lambda_{0} \mid \alpha_{i}\right.$ is a long root of $\psi$, $1 \leqslant i \leqslant k\}$. The set $\psi_{l}^{+}$of positive long roots of $\psi$ is a set of strongly orthogonal roots of noncompact type and $\lambda \rightarrow \kappa_{\lambda}(v)$ is the trivial character of $\Lambda_{\psi} / \Lambda_{0}$ if and only if $v^{-1} \alpha \equiv \alpha$ for all $\alpha \in \psi_{l}^{+}$. This is the case if $v \in U(\psi) W_{K}$. Conversely, if $v^{-1} \alpha \equiv \alpha$ for all $\alpha \in \psi_{l}^{+}$, then $\psi_{l}^{+}$and $v^{-1} \psi_{l} \cap \Phi^{+}$are two sets of strongly orthogonal roots of noncompact type in $\Phi$, and hence conjugate by an element of $W_{K}$. Thus $w v^{-1} \psi_{l}=\psi_{l}$ for some $w \in W_{K}$ and so $v \in U W_{K}$ where $U=\left\{u \in W \mid u \psi_{l}=\psi_{l}\right\}$. In all cases, $U W_{K}=U(\psi) W_{K}$. Finally, note that $W_{1}\left(\Phi: \psi^{+}\right) \subseteq W_{K}$ so that $[U(\psi) \cap$ $\left.W_{K}\right]=\left[S(\psi) \cap W_{K}\right]\left[W_{1}\left(\Phi: \psi^{+}\right)\right]$and $\left[S(\psi) \cap W_{K}\right]=\left[L_{\psi}: L\right]$. Thus

$$
\begin{aligned}
\sum_{\lambda \in \Lambda_{1}(\psi)} c(\lambda)^{-1}\left[W_{K}\right]^{-1} & \sum_{w \in W\left(M^{0}, H_{K}^{0}\right)} \operatorname{det} w \overline{b^{*}\left(\gamma h_{0}(w)\right)} \\
& \times \sum_{v \in W} \operatorname{det} v \kappa_{\lambda}(v) P\left(\psi^{+}, \mu, \gamma v h_{1}(w)\right) \\
\equiv & \varepsilon\left(\psi: \Phi^{+}\right)\left[W_{1}\left(\Phi: \psi^{+}\right)\right]^{-1}\left[L_{\psi}: L_{R}\right]^{-1} \\
& \times \sum_{w \in W\left(M^{0}, H_{K}^{0}\right)} \operatorname{det} w \overline{b^{*}\left(\gamma h_{0}(w)\right)} P\left(\psi^{+}, \mu, \gamma h_{1}(w)\right) .
\end{aligned}
$$

If $\psi$ is of type $A_{1}^{n}$, then $\Lambda_{1}(\psi)=\Lambda_{1}$ and $P\left(\psi^{+}, \mu, \gamma h_{1}(w)\right)=Q\left(\psi^{+}, \mu, \gamma h_{1}(w)\right)$ so the proof is complete.

Case II. Suppose $\varphi \subsetneq \psi$ and $\Phi$ is simple of type $B_{2 s}, s \geqslant 1$, or $F_{2 s}, s=2$. Then $\psi$ is of type $B_{2}^{s}$ and $\varphi$ is of type $B_{2}^{s-1} \times B_{1}^{2}$. Then $\left\{\lambda+\Lambda_{0} \mid \varphi \subseteq \Phi(\lambda)\right\}$ is the disjoint 
union of $\left\{\lambda+\Lambda_{0} \mid \varphi \in \mathcal{T}(\Phi(\lambda))\right\}$ and $\left\{\lambda+\Lambda_{0} \mid \psi \subseteq \Phi(\lambda)\right\}$. Thus

$$
\sum_{\lambda+\Lambda_{0}+\varphi \in \mathcal{T}(\Phi(\lambda))} \kappa_{\lambda}(v)=\sum_{\lambda \in \Lambda_{\varphi} / \Lambda_{0}} \kappa_{\lambda}(v)-\sum_{\lambda \in \Lambda_{\psi} / \Lambda_{0}} \kappa_{\lambda}(v)
$$

where, as before, $\Lambda_{\varphi}=\{\lambda \in \Lambda \mid \varphi \subseteq \Phi(\lambda)\}$. Now $U(\varphi) W_{K}=U(\psi) W_{K}$ so that if $v \notin U(\psi) W_{K}, \lambda \rightarrow \kappa_{\lambda}(v)$ is nontrivial on $\Lambda_{\psi}$ and hence also on $\Lambda_{\varphi} \supseteq \Lambda_{\psi}$ and so $\Sigma_{\lambda+\Lambda_{0} \rightarrow \varphi \in \mathcal{T}(\Phi(\lambda))} \kappa_{\lambda}(v)=0$. The double coset $U(\varphi) W_{K}$ of $U(\varphi) \backslash W / W_{K}$ can be represented by $v=1$ so that

$$
\sum_{\lambda+\Lambda_{0} \dagger \varphi \in \mathcal{T}(\Phi(\lambda))} \kappa_{\lambda}(1)=\left[L_{\varphi}: L\right]-\left[L_{\psi}: L\right]=\left[L_{\psi}: L\right] .
$$

Further, $\left[L_{\psi}: L\right] /\left[U(\varphi) \cap W_{K}\right]\left[L_{\varphi}: L_{R}\right]=s /\left[W_{1}\left(\Phi: \psi^{+}\right)\right] 4\left[L_{\psi}: L_{R}\right]$ so that

$$
\begin{aligned}
\sum_{\lambda \in \Lambda_{1}(\varphi)} c(\lambda)^{-1}\left[W_{K}\right]^{-1} & \sum_{w \in W\left(M^{0}, H_{K}^{0}\right)} \operatorname{det} w \overline{b^{*}\left(\gamma h_{0}(w)\right)} \\
& \times \sum_{v \in W} \operatorname{det} v \kappa_{\lambda}(v) P\left(\varphi^{+}, \mu, \gamma v h_{1}(w)\right) \\
\equiv & s / 4\left[W_{1}\left(\Phi: \psi^{+}\right)\right]^{-1}\left[L_{\psi}: L_{R}\right]^{-1} \varepsilon\left(\psi: \Phi^{+}\right) \\
& \times \sum_{w \in W\left(M^{0}, H_{K}^{0}\right)} \operatorname{det} w \overline{b^{*}\left(\gamma h_{0}(w)\right)} P\left(\varphi^{+}, \mu, \gamma h_{1}(w)\right) .
\end{aligned}
$$

In this case $\Lambda_{1}=\Lambda_{1}(\psi) \cup \Lambda_{1}(\varphi)$. If $E$ is defined as in (4.5) with respect to $\psi=\varphi_{1} \cup \cdots \cup \varphi_{s}$, then there is $1 \leqslant j \leqslant s$ so that

$$
P\left(\varphi^{+}, \mu, h\right)=\sum_{\left(\eta_{1}, \ldots, \eta_{s}\right) \in E} \prod_{\substack{i=1 \\ i \neq j}}^{s} S\left(\varphi_{i}^{+}, \mu_{i}, \eta_{i} h_{i}\right) \times S\left(\varphi_{j, s}^{+}, \mu_{j}, \eta_{j} h_{j}\right)
$$

for all $\mu \in \mathrm{i}_{p}^{*}, h \in \exp \left(i \mathrm{i}_{p}\right)$. Further, the $s$ possible $\varphi$ 's which could have been chosen, corresponding to $1 \leqslant j \leqslant s$, are all conjugate by elements of determinant one in $W_{K} \subseteq \nu W\left(M^{0}, H_{K}^{0}\right)$. Thus

$$
\begin{aligned}
& \sum_{w \in W\left(M^{0}, H_{K}^{0}\right)} \operatorname{det} w \overline{b^{*}\left(\gamma h_{0}(w)\right)} I_{J}^{H}\left(\gamma: h_{1}(w): \mu\right) \\
& \equiv(\pi i)^{n}\left[W_{1}\left(\Phi: \psi^{+}\right)\right]^{-1}\left[L_{\psi}: L_{R}\right]^{-1} \varepsilon\left(\psi: \Phi^{+}\right) \sum_{w \in W\left(M^{0}, H_{K}^{0}\right)} \operatorname{det} w \overline{b^{*}\left(\gamma h_{0}(w)\right)} \\
& \quad \times \sum_{\left(\eta_{1}, \ldots, \eta_{s}\right) \in E} \sum_{j=1}^{s} \prod_{\substack{i=1 \\
i \neq j}}^{s} S\left(\varphi_{i}^{+}, \mu_{i}, \eta_{i} h_{i}\right) \times T\left(\varphi_{j}^{+}, \mu_{j}, \eta_{j} h_{j}\right) .
\end{aligned}
$$

The lemma follows because any term in $Q\left(\psi^{+}, \mu, h\right)$ which contains a factor of the form $S\left(\varphi_{i, s}^{+}, \mu_{i}, h_{i}\right) S\left(\varphi_{j, s}^{+}, \mu_{j}, h_{j}\right)$ for some $1 \leqslant i \neq j \leqslant s$ is stable under $h \rightarrow v h$, where $v$ is an element of $W_{K}$ of determinant -1 and thus will cancel out in the sum over $W\left(M^{0}, H_{K}^{0}\right)$.

Case III. Suppose $\Phi$ is simple of type $B_{2 s+1}$. Then $\psi=\varphi_{1} \cup \cdots \cup \varphi_{s+1}$ is of type $B_{2}^{s} \times B_{1}$ and $\Lambda_{1}(\psi)=\Lambda_{1}$. The lemma follows from Case I together with the observation that any term in $Q\left(\psi^{+}, \mu, h\right)$ which contains a factor of the form 
$S\left(\varphi_{i, s}^{+}, \mu_{i}, h_{i}\right) S\left(\varphi_{j}^{+}, \mu_{j}, h_{j}\right)$ for $i, j$ with $\varphi_{i}$ of type $B_{2}$ and $\varphi_{j}$ of type $B_{1}$ is stable under $h \rightarrow v h$ for an element $v \in W_{K}$ with det $v=-1$.

Case IV. Suppose $\varphi \subsetneq \psi$ and $\Phi$ is simple of type $C_{n}$. Then $\psi$ is of type $C_{2}^{k}$ or $C_{2}^{k} \times C_{1}$ depending on whether $n=2 k$ or $2 k+1$, and $\varphi$ is of type $A_{1}^{2 p} \times C_{2}^{k-p}$ or $A_{1}^{2 p} \times C_{2}^{k-p} \times C_{1}$ for some $0<p \leqslant k$ where the $A_{1}$ roots are short. In any case, $U(\varphi) W_{K}=U(\varphi) W_{K}=W$, so the only double coset of $U(\varphi) \backslash W / W_{K}$ can be represented by $v=1$. Also in this case, for a given $\varphi$, there is only one coset of $\Lambda / \Lambda_{0}$ with $\varphi \in \mathcal{T}(\Phi(\lambda))$. Finally, $\left[U(\psi) \cap W_{K}\right]=2^{-p}\left({ }_{p}^{k}\right)\left[U(\varphi) \cap W_{K}\right]$ and $\left[L_{\varphi}: L_{\psi}\right]=2^{p}$ so that

$$
\begin{aligned}
\sum_{\lambda \in \Lambda_{1}(\varphi)} c(\lambda)^{-1}\left[W_{K}\right]^{-1} & \sum_{w \in} \begin{array}{l} 
\\
\end{array} \\
& \times \sum_{v \in W} \operatorname{det} w \overline{b^{*}\left(\gamma h_{0}^{0}\right)} \operatorname{det} v \kappa_{\lambda}(v) P\left(\varphi^{+}, \mu, \gamma v h_{1}(w)\right) \\
\equiv & \left(\begin{array}{l}
k \\
p
\end{array}\right) 4^{-p}\left[W_{1}\left(\Phi: \psi^{+}\right)\right]^{-1}\left[L_{\psi}: L_{R}\right]^{-1} \varepsilon\left(\psi: \Phi^{+}\right) \\
& \times \sum_{w \in W\left(M^{0}, H_{K}^{0}\right)} \operatorname{det} w \overline{b^{*}\left(\gamma h_{0}(w)\right)} P\left(\varphi^{+}, \mu, \gamma h_{1}(w)\right) .
\end{aligned}
$$

Now, if $E$ is defined as in (4.5) with respect to $\psi=\varphi_{1} \cup \cdots \cup \dot{\varphi}_{s}, s=k$ or $k+1$ depending on whether $\Phi$ is of type $C_{2 k}$ or $C_{2 k+1}$, there is a subset $P$ of $\{1, \ldots, s\}$ with $p$ elements so that

$$
P\left(\varphi^{+}, \mu, h\right)=\sum_{\left(\eta_{1}, \ldots, \eta_{s}\right) \in E} \prod_{i \notin P} S\left(\varphi_{i}^{+}, \mu_{i}, \eta_{i} h_{i}\right) \prod_{i \in P} S\left(\varphi_{i, s}^{+}, \mu_{i}, \eta_{i} h_{i}\right) .
$$

For fixed $p, 0 \leqslant p \leqslant k$, there are $\left(\begin{array}{l}k \\ p\end{array}\right)$ such $\varphi$ which could have been chosen all conjugate by elements of determinant one in $W_{K}$. Thus

$$
\begin{aligned}
\sum_{w \in W\left(M^{0}, H_{K}^{0}\right)} \operatorname{det} w \overline{b^{*}\left(\gamma h_{0}(w)\right)} I_{J}^{H}\left(\gamma: h_{1}(w): \mu\right) & \\
\equiv & (\pi i)^{n}\left[W_{1}\left(\Phi: \psi^{+}\right)\right]^{-1}\left[L_{\psi}: L_{R}\right]^{-1} \varepsilon\left(\psi: \Phi^{+}\right) \\
& \times \sum_{w \in W\left(M^{0}, H_{K}^{0}\right)} \operatorname{det} w \overline{b^{*}\left(\gamma h_{0}(w)\right)} Q\left(\psi^{+}, \mu, \gamma h_{1},(w)\right) .
\end{aligned}
$$

This concludes the proof of the lemma for $\Phi^{+}$chosen as above.

Now suppose $\Phi^{+}$is replaced by $v \Phi^{+}, v \in W(\Phi)$. The the left-hand side of (4.9) changes by det $v$. Write $v=u \sigma^{-1}$ where $u \in W(\psi)$ and $\sigma \psi^{+} \subseteq \Phi^{+}$. Then

$$
\varepsilon\left(\psi: v \Phi^{+}\right)=\varepsilon\left(v^{-1} \psi: \Phi^{+}\right)=\operatorname{det} \sigma \varepsilon\left(\psi: \Phi^{+}\right) .
$$

Further, $\psi \cap v \Phi^{+}=u \psi^{+}$and

$$
\begin{gathered}
\sum_{w \in W\left(M^{0}, H_{K}^{0}\right)} \operatorname{det} w \overline{b^{*}\left(\gamma h_{0}(w)\right)} Q\left(u \psi^{+}, \mu, \gamma h_{1}(w)\right) \\
\equiv \operatorname{det} u \sum_{w \in W\left(M^{0}, H_{K}^{0}\right)} \operatorname{det} w \overline{b^{*}\left(\gamma h_{0}(w)\right)} Q\left(\psi^{+}, \mu, \gamma h_{1}(w)\right) .
\end{gathered}
$$


Thus (4.9) is valid for any choice of positive root system for $\Phi$.

We now have, using (4.9), a complete description of the terms $I_{J}^{H}(\gamma: j: \mu)$ which appear in Theorem 4.3. To complete the proof of Theorem 1, it is only necessary to use this information to compute the terms

$$
\sum_{S \in \delta(H, J)}(-1)^{l} I\left(S: b^{*}: \mu: h_{k}\right)
$$

appearing in (4.3). This stage of the proof can be accomplished in the same way as the corresponding analysis in [5d,e]. It is slightly more complicated for three reasons.

First, comparing the formulas for $I_{J}^{H}$ to those for $\bar{I}_{J}^{H}$ in [5d, e], we see that the $I_{J}^{H}$ may have extra terms. These will be carried along at each stage and cause no extra difficulties. Second, in computing the Fourier inversion formula for $F_{f}^{H}\left(h_{k} h_{p}\right)$, many sums of the form $\Sigma_{w \in W\left(M^{0}, H_{K}^{0}\right)}$ det $w g\left(b^{*}, \mu, w h_{k}\right)$ appear, where $g$ is some function of $b^{*} \in \hat{J}_{K}, \mu \in \mathrm{i}_{p}^{*}$, and $h_{k} \in H_{K}$ where $J \in \operatorname{Car}^{\prime}(M)$. In [5d], the corresponding terms would be averaged over the larger Weyl group $W_{I}=W_{I}(\mathfrak{g}, \mathfrak{h})=W\left(\mathfrak{m}_{\mathbf{C}}, \mathfrak{h}_{k \mathbf{C}}\right)$. Frequently $\sum_{w \in W_{I}}$ det $w g\left(b^{*}, \mu, w h_{k}\right) \equiv 0$, while $\sum_{w \in W\left(M^{0}, H_{K}^{0}\right)} \operatorname{det} w g\left(b^{*}, \mu, w h_{k}\right)$ makes a nonzero contribution to the Fourier inversion formula for $F_{f}^{H}$. Thus in passing from $I_{J}^{H}(\gamma: j: \mu)$ to $\Sigma_{\gamma \in \Gamma\left(\mathrm{i}_{p}\right)} b^{*}(\gamma) I_{J}^{H}(\gamma: j: \mu)$ and on to

$$
\sum_{S \in \delta(H, J)}(-1)^{l} I\left(S: b^{*}: \mu: h_{k}\right),
$$

terms which would make no contribution to the Fourier inversion formula for an averaged invariant integral, need to be retained in the formula for $F_{f}^{H}$. However, they can be handled by the same techniques used in [5d].

Finally, in [5d, e] we restricted ourselves to the case that $H=T$ is a compact Cartan subgroup of $G$. In this paper, we drop that simplifying assumption. For the most part, this causes no problems. For example, for $J \in \operatorname{Car}^{\prime}(M), \delta(H, J)$ can be described in the same way as $\delta(J)$ in [5d], where $M^{0}$ takes the role of $G$ and $H_{K}^{0}$ that of $T$. For a fixed $S \in \mathcal{S}(H, J)$, the constants picked up in evaluating

$$
\prod_{i=1}^{l} w_{i} \sum_{\gamma_{i} \in \Gamma\left(a_{i}\right)} b^{*}\left(\gamma_{i}\right) \tilde{I}_{J_{i}}^{\tilde{i_{i-1}}}\left(\gamma_{i}: h_{i}: \mu_{i}\right)
$$

are the same as those occurring in [5d, e], except that if $Z\left(\mathfrak{h}_{p}\right) \cap Z\left(\dot{i}_{p}\right)$ is not trivial, the extra term $\left[Z\left(\mathfrak{h}_{p}\right) \cap Z\left(\dot{\mathfrak{d}}_{p}\right)\right]^{-1}$ factors out. In this way, we obtain the following.

Lemma 4.10. Let $J \in \operatorname{Car}(M)$. Then

$$
\begin{aligned}
& \frac{(-1)^{r_{I}(H)}}{(4 \pi)^{\operatorname{dimi} i_{p}}\left[W\left(M_{J}, J_{K}\right)\right]} \sum_{w \in W\left(M^{0}, H_{K}^{0}\right)} \operatorname{det} w \sum_{S \in \delta(H, J)}(-1)^{l} I\left(S: b^{*}: \mu: w h_{k}\right) \\
& \equiv \frac{(-1)^{r_{l}(J)}}{[W(M, J)]\left[Z\left(\mathfrak{h}_{p}\right) \cap Z\left(\dot{\mathrm{i}}_{p}\right)\right]}\left(\frac{\mathrm{i}}{2}\right)^{\operatorname{dimi}_{p}} \\
& \times \sum_{w \in W\left(M^{0}, H_{K}^{0}\right)} \operatorname{det} w K\left(M, J, b^{*}, \mu, w h_{k}\right) .
\end{aligned}
$$


To complete the proof of Theorem 1 it is necessary to make two observations. First, representatives $w$ of $W\left(M, H_{K}\right) / W\left(M^{0}, H_{K}^{0}\right)$ can be chosen so that for all $h_{k} \in H_{K}, K\left(M, J, b^{*}, \mu, w h_{k}\right) \equiv \operatorname{det} w K\left(M, J, b^{*}, \mu, h_{k}\right)$. Thus

$$
\begin{array}{r}
{\left[W\left(M, H_{K}\right): W\left(M^{0}, H_{K}^{0}\right)\right] \sum_{w \in W\left(M^{0}, H_{K}^{0}\right)} \operatorname{det} w K\left(M, J, b^{*}, \mu, w h_{k}\right)} \\
\quad \equiv \sum_{w \in W\left(M, H_{K}\right)} \operatorname{det} w K\left(M, J, b^{*}, \mu, w h_{k}\right) .
\end{array}
$$

Second, although Theorem 4.3 is valid only for $h$ in the dense open subset $H^{*}$ of $H^{\prime}$, both sides of the equation in Theorem 1 are continuous functions of $h \in H^{\prime}$. Thus the formula is true for all $h \in H^{\prime}$.

\section{REFERENCES}

1. D. Barbasch, Fourier inversion for unipotent invariant integrals, Trans. Amer. Math. Soc. 249 (1979), $51-83$.

2. W. Chao, Fourier inversion and the Plancherel formula for semisimple Lie groups of real rank two, $\mathrm{Ph}$. D. Thesis, Univ. of Chicago, Chicago, Ill., 1977.

3. Harish-Chandra, (a) Discrete series for semisimple Lie groups. I, Acta Math. 113 (1965), 241-318. (b) Two theorems on semi-simple Lie groups, Ann. of Math. (2) 83 (1966), 74-128. (c) Supertempered distributions on real reductive groups, 1979 (preprint).

4. H. Hecht, The characters of Harish-Chandra representations, Math. Ann. 219 (1976), 213-226.

5. R. Herb, (a) Character formulas for discrete series on semisimple Lie groups, Nagoya Math. J. 64 (1976), 47-61. (b) Fourier inversion of invariant integrals on semisimple real Lie groups, Trans. Amer. Math. Soc. 249 (1979), 281-302. (c) Characters of averaged discrete series on semisimple real Lie groups, Pacific J. Math. 80 (1979), 169-177. (d) Fourier inversion and the Plancherel theorem for semisimple real Lie groups, Amer. J. Math. 104 (1982), 9-58. (e) Fourier inversion and the Plancherel theorem, (Proc. Marseille Conf., 1980), Lecture Notes in Math., vol. 880, Springer-Verlag, Berlin and New York, 1982, pp. 197-210.

6. R. Herb and P. Sally, Singular invariant eigendistributions as characters in the Fourier transform of invariant distributions, J. Funct. Anal. 33 (1979), 195-210.

7. T. Hirai, Invariant eigendistributions of Laplace operators on real simple Lie groups. IV: Characters of discrete series for $\mathrm{Sp}(n, \mathbf{R})$, Japan J. Math. 3 (1977), 1-48.

8. S. Martens, The characters of the holomorphic discrete series, Proc. Nat. Acad. Sci. U.S.A. 72 (1975), 3275-3276.

9. H. Midorikawa, On the explicit formulae of characters for discrete series representations, Japan J. Math (N.S.) 3 (1977), 313-368.

10. P. Sally and G. Warner, The Fourier transform on semisimple Lie groups of real rank one, Acta Math. 131 (1973), 1-26.

11. W. Schmid, On the characters of discrete series, Invent. Math. 30 (1975), 47-144.

12. D. Shelstad, (a) Orbital integrals and a family of groups attached to a real reductive group, Ann. Sci. École Norm. Sup. 12 (1979), 1-31. (b) Embeddings of L-groups (preprint). (c) L-indistinguishability for real groups (preprint).

13. J. Vargas, $A$ character formula for the discrete series of a semisimple Lie group, Bull. Amer. Math. Soc. 2 (N.S.) (1980), 465-467.

14. G. Warner, Harmonic analysis on semisimple Lie groups, Vols. I, II, Springer-Verlag, Berlin and New York, 1972.

Department of Mathematics, University of Maryland, College Park, Maryland 20742 